\title{
Adoption of the Internet of Things (IoT) in Agriculture and Smart Farming towards Urban Greening: A Review
}

\author{
A. A. Raneesha Madushanki ${ }^{1}$, Malka N Halgamuge ${ }^{2}$, W. A. H. Surangi Wirasagoda ${ }^{3}$, Ali Syed ${ }^{4}$ \\ School of Computing and Mathematics, Charles Sturt University, Melbourne, Australia ${ }^{1,3,4}$ \\ Department of Electrical and Electronic Engineering, The University of Melbourne ${ }^{2}$
}

\begin{abstract}
It is essential to increase the productivity of agricultural and farming processes to improve yields and costeffectiveness with new technology such as the Internet of Things (IoT). In particular, IoT can make agricultural and farming industry processes more efficient by reducing human intervention through automation. In this study, the aim to analyze recently developed IoT applications in the agriculture and farming industries to provide an overview of sensor data collections, technologies, and sub-verticals such as water management and crop management. In this review, data is extracted from 60 peer-reviewed scientific publications (20162018) with a focus on IoT sub-verticals and sensor data collection for measurements to make accurate decisions. Our results from the reported studies show water management is the highest subvertical $(28.08 \%)$ followed by crop management $(14.60 \%)$ then smart farming $(10.11 \%)$. From the data collection, livestock management and irrigation management resulted in the same percentage $(5.61 \%)$. In regard to sensor data collection, the highest result was for the measurement of environmental temperature $(24.87 \%)$ and environmental humidity (19.79\%). There are also some other sensor data regarding soil moisture $(15.73 \%)$ and soil pH $(\mathbf{7 . 6 1 \%})$. Research indicates that of the technologies used in IoT application development, Wi-Fi is the most frequently used $(30.27 \%)$ followed by mobile technology $(21.10 \%)$. As per our review of the research, we can conclude that the agricultural sector $\mathbf{( 7 6 . 1 \% )}$ is researched considerably more than compared to the farming sector $(\mathbf{2 3 . 8 \%})$. This study should be used as a reference for members of the agricultural industry to improve and develop the use of IoT to enhance agricultural production efficiencies. This study also provides recommendations for future research to include IoT systems' scalability, heterogeneity aspects, IoT system architecture, data analysis methods, size or scale of the observed land or agricultural domain, IoT security and threat solutions/protocols, operational technology, data storage, cloud platform, and power supplies.
\end{abstract}

Keywords-Internet of Things; IoT; agricultural; smart farming; business; sensor data; automation

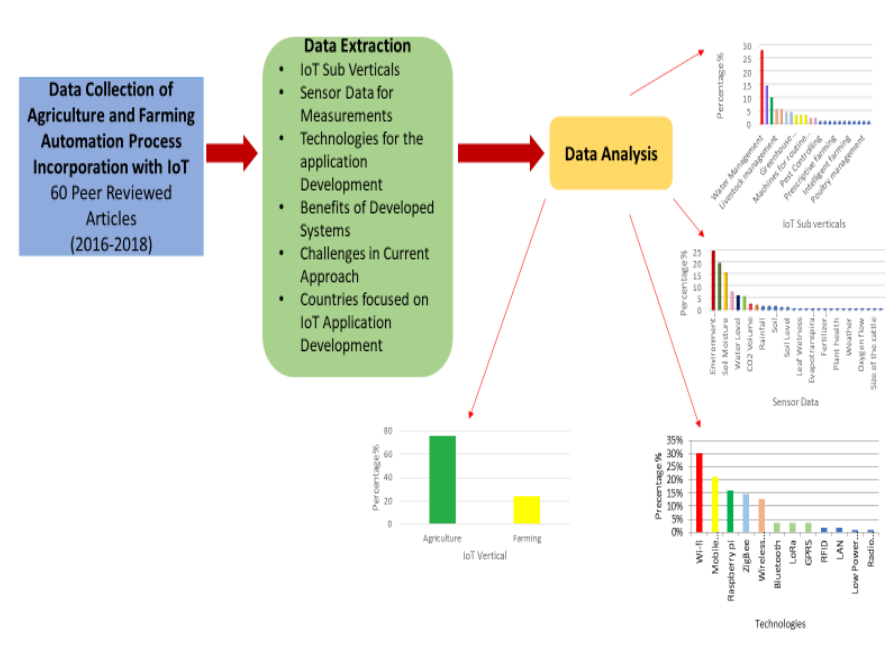

Graphical Abstract.

\section{INTRODUCTION}

IoT is a combination of worldwide data, web associated items or things, and is an integral component of the future Internet. IoT focuses on the automation of processes by lessening human interaction. In the process of automation, IoT collects data using sensors and processes the data using controllers and completing the automation processes by using actuators [1], [2]. IoT in agriculture and farming focus is on automating all the aspects of farming and agricultural methods to make the process more efficient and effective. Traditional approaches in livestock management (such as cattle detection) are not fully automated and have many inefficiencies such as higher human interaction, labour cost, power consumption, and water consumption [1], [3], [4], [5], [6]. The central concept of this review is to analyse the IoT sub-verticals, collected data for measurements and used technologies to develop applications. It is essential to identify the most researched sub-verticals, data collections and technologies to create new IoT applications in the future. 
This review provides an overall picture of currently developed IoT applications in agriculture and farming between 2016 and 2018.

As a solution to the existing problems, researchers have focused on smart agricultural and farming automated systems with the help of IoT [7], [8], [9], [10]. IoT is the network of things which identifies elements clearly with the help of software intelligence, sensors and ubiquitous connectivity to the Internet. In IoT, the data that collects from Internetconnected items or things contains with gadgets, sensors and actuators [1]. Many researchers have focused on smart systems for monitoring and controlling agricultural parameters by enhancing productivity and efficiency. Smart systems collect data for measurements to get accurate results that can lead to appropriate actions. Current use of smart agricultural systems relates to collecting data on environmental parameters such as temperature, humidity, soil moisture and $\mathrm{pH}$ [11], [12], [13]. With accurate sensor data collection using a range of different sensors, researchers have implemented smart agricultural systems to make the farm process more effective [9], [14]. Research has mainly focused on sub-verticals such as water management, crop management and smart farming to make processes automated by reducing human intervention, costs, power consumption and water consumption.

The automation process of agricultural and farming reduced human interaction and improve the efficiency. The reason for that is every country population depends on agriculture thus consumers of these resources should use water and land resources optimally [19], [20]. Moreover, it is imperative to have good quality production and crop management in order to maximize profitability. Hence, IoT base agricultural management systems are integral for an agriculturally based country. The new systems developed using IoT technologies have reduced the drawbacks associated with traditional approaches and provided many advantages to farmers. For example, IoT-based water management systems collect environmental attributes such as temperature, water level and humidity through the sensors and provide accurate irrigation timing [19], [21]. In addition, crop management systems developed using IoT monitor the temperature, humidity and soil through sensors thus providing adequate information so that farmers can manage the crops appropriately [25]. Overall, these IoT-based systems help to reduce human interaction, power utilization and reduce cost in the field of agriculture. Moreover, IoT-based agricultural related applications have been used in the area of pest control, weather monitoring, nutrient management and greenhouse management.

IoT for agriculture uses sensors to collect big data on the agricultural environment. It discovers, analyses and deals with models built upon big data to make the development of agriculture more sustainable [34]. IoT can provide efficient and low-cost solutions to the collection of data. Weather, Water Scarcity, Soil fertility and Pesticides are the significant players in it. IoT will make agriculture beneficiary. Agriculture and farming depend on water [35]. Farmers depend on rainfall for all their agricultural needs.
Fertilizer also plays a very significant role in the field of agriculture by helping to increase the productivity of plants [36]. By using IoT, farmers can manage soil condition more effectively and at less expense by monitoring them from any location [37]. The primary objective of this study is how IoT and technologies are used in conserving water, fertiliser and energy in the agricultural industry by combining new technologies. This has benefits for the development of the economy of countries as well as the wealth of the people [38]. With the combination of both advanced technologies in hardware and software, IoT can track and count all relevant aspects of production which can reduce the waste, loss and cost [39]. The information needed to make smart decisions can be obtained merely by using electronic devices [40]. IoT transforms the agricultural industry and enables farmers to overcome different challenges. Innovative applications can address these issues and therefore increase the quality, quantity, sustainability and cost-effectiveness of crop production [41], [42], [43]. IoT provides more benefits to the farming industry by improving the health of animals through better food and environment, addressing the labour shortage issue as well cost savings through automation, increase in milk production, and increase in some animals during the breeding period through detection of estrus cycle and additional revenue streams from waste.

Our study has analyzed recently developed IoT applications in the fields of agriculture and farming to address current issues such as unnecessary human interaction leading to higher labour cost, unnecessary water consumption and water-saving measures for the future, higher energy consumption, energy-saving measures for the future and crop monitoring difficulties. According to our analysis, we can identify a focus on water and crop management as subverticals in the agriculture and farming sectors. This survey also focusses on other agriculture and farming sub-verticals to identify the gap between IoT application developments in the least researched areas. The IoT generates enormous data, socalled big data (high volume, at a different speed and different varieties of data) in varying data quality. Analysing the IoT system and its key attributes are the key to advancing smart IoT utilization. Therefore, the primary aim of our paper is to explore recently created IoT applications in the agriculture and farming industry to give the more profound understanding about sensor data collection, used technologies, and subverticals, for example, water and crop management. The secondary aim of this study is to analyse the current issues such as higher human interaction, high labour cost, higher water consumption and save water for future, higher energy consumption and save energy/electricity for future, crop monitoring difficulties in IoT for agriculture and farming.

The remainder of this paper is as follows: In Section II we include raw data collection methodology, data inclusion criteria, and data analysis methods. Finally, the results of Agriculture and Farming based on IoT Sub verticals, Sensor Data, and Technologies are presented in Section III, and in Section IV we discuss the results. Section V concludes the paper. The raw data collected from 60 peer-reviewed publications used in this paper are summarised in Table I. 


\section{MATERIALS AND METHODS}

Data collection involves identifying important criteria in research articles on the Internet of Things (IoT) in the agriculture and farming sectors.

As shown in Table I, these essential criteria were used to analyse relevant research papers. In particular, 60 peerreviewed scientific publications on IoT in the agriculture and farming sectors published in scientific journals between 2016 and 2018 were used.

1) Collection of raw data: The data gathered for this review is from 60 peer-reviewed publications (2016-2018) that were collected from the IEEE database. All these publications have different data applications that have been studied and analyzed in this survey. The attributes compared were sub-verticals, data collection measurements, used technologies, challenges in current approach, benefits, countries and drivers of IoT.

2) Data inclusion criteria: To evaluate the data inclusion criteria a comparison table was drawn to include as the following attributes: Author, Sub vertical, Data collection measurements, Technologies, Benefits, Challenges, Solutions and Drivers of IoT. Nevertheless, in our study, articles were excluded when the selected attributes were not present. In our analysis, the number of sensors, amount of data collected, underlying technologies, sensor topology and other intermediate gateways were not included since no information can find with all the peer-reviewed publications (2016-2018).

3) Data analysis: We pooled and analyzed the reported studies based on data collected through peer reviewed articles and displaying emerging themes in a table. The data sets included attributes such as Sub vertical, Data collection measurements, Technologies, Benefits, Challenges, Solutions, Countries focused on automation of the agriculture proses and Drivers of IoT. The descriptive details of the study based on the publication year were analyzed to observe the results from 2016 to 2018.

\section{RESULTS}

This review aims to analyse the incorporation of IoT for the development of applications in the agriculture and farming sectors. The study focuses on sub-verticals and collecting data for measurements and technologies in the field of agriculture and farming to increase productivity and efficiency with the help of the Internet of Things (IoT). This study of IoT in agriculture and farming focuses on developing a criterion approach with the help of agricultural environmental parameters and IoT measures and technologies. In the field of agriculture, there are many environmental parameters that need to be considered to enhance crops, reduce water consumption and human involvement [44]. Moreover, there are many sub-verticals that can be identified depending on the differences in approach.

In this review, we have gathered articles which have focused on agricultural and farming sub-verticals from 2016 to 2018. As shown in Fig. 1, 23 sub-verticals were found according to the results obtained and the topmost area was water management $(28.08 \%)$.

As IoT depends on sensor data collections, a vast amount of data needs to be gathered to identify or predict accurate results. This study indicates that many researchers have focused on environmental temperature (24.87\%), humidity $(19.79 \%)$ and soil moisture $(15.73 \%)$ as environmental measurements. As shown in Fig. 2, 28 types of data were collected for measurements with environmental temperature and humidity being considered the most critical parameters for agriculture and farming.

As shown in Fig. 3, we have categorised all technologies used in the articles. This study has identified Wi-Fi as the most used technology $(30.27 \%)$ followed by Mobile Technology $(21.10 \%)$ for both agriculture and farming. ZigBee, another data transfer technology, is also used but to a lesser extent.

According to Fig. 4, the use of IoT was more prominent in the agriculture industry than the farming industry (Agriculture $-76.1 \%$, Farming $-23.8 \%$ ).

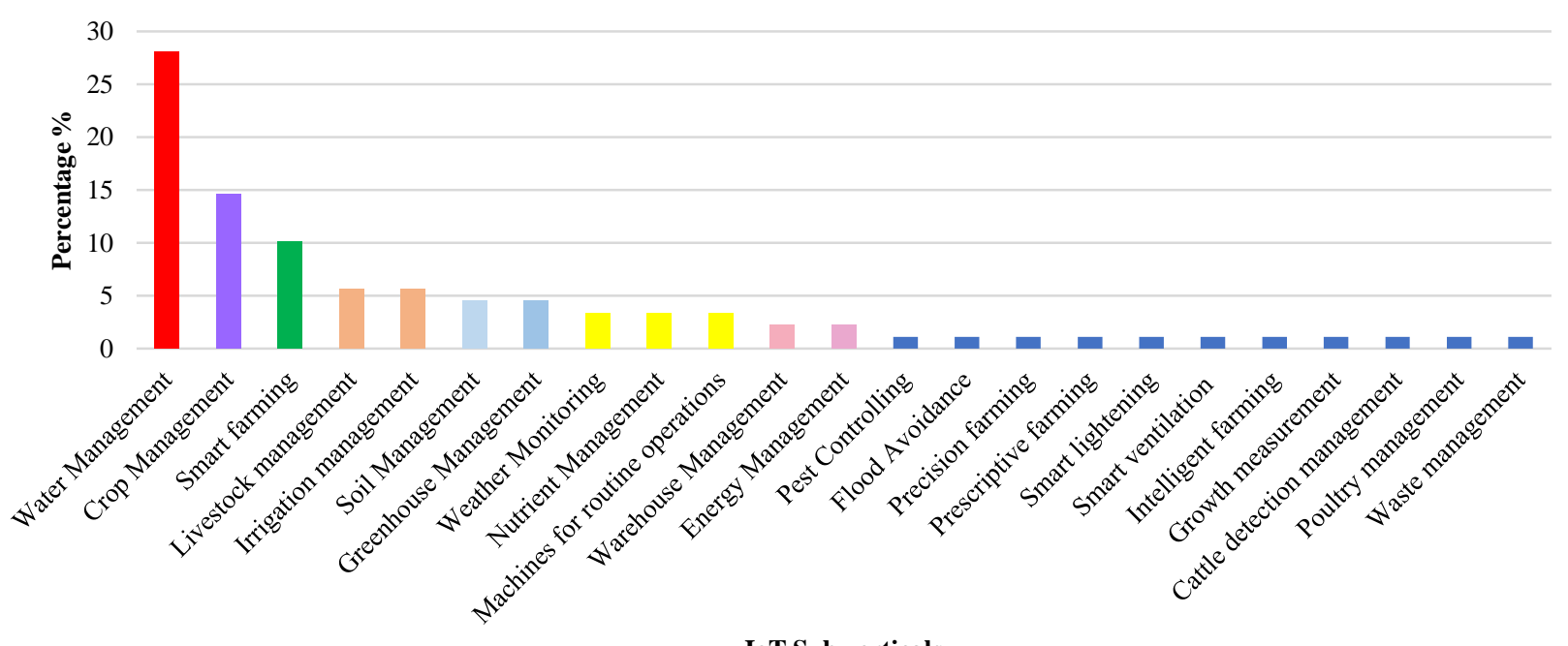

IoT Sub verticals

Fig. 1. Agriculture and Farming Sub Verticals: different Agricultural and Farming Sub Verticals Considered to Enhance Efficiency and Productivity-Pooling 
Data from the 60 Scientific Peer-Reviewed Publications Published in 2016-2018.

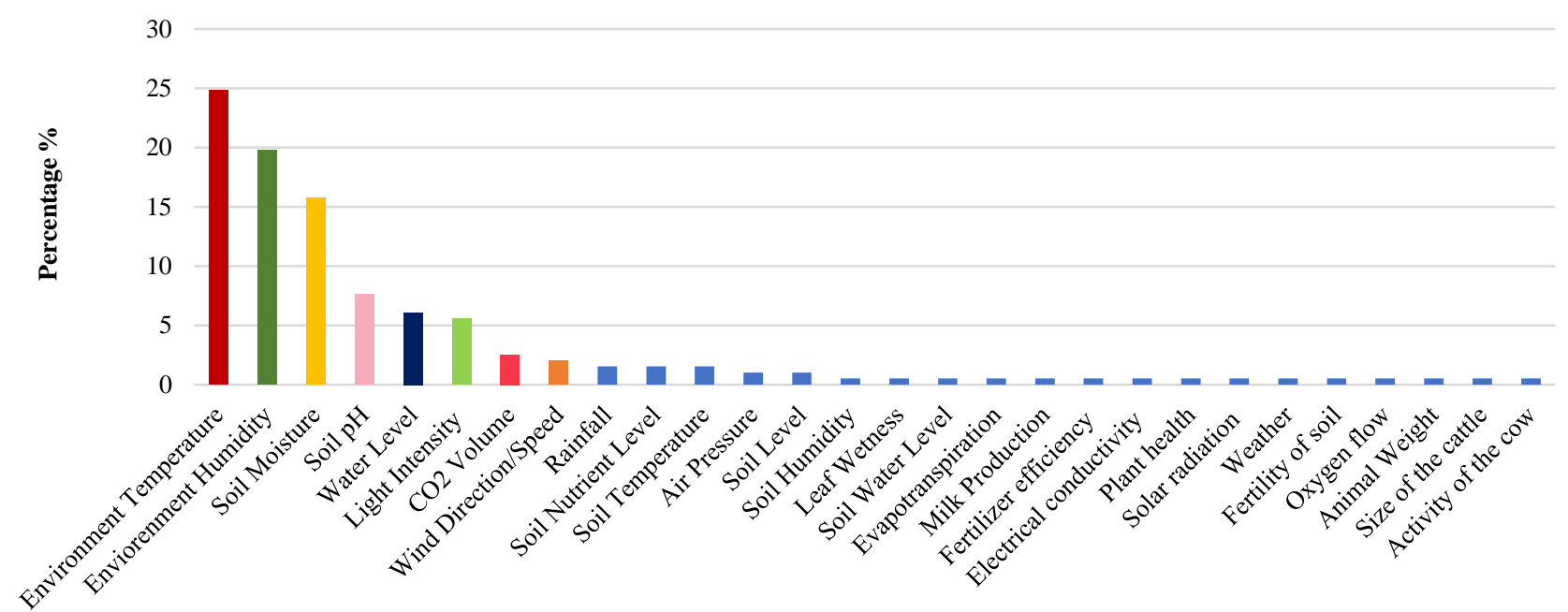

Sensor Data

Fig. 2. Utilization of Sensor Data based on Farming Activities Referred to in the Data Pool of 60 Peer Reviewed Published Articles.

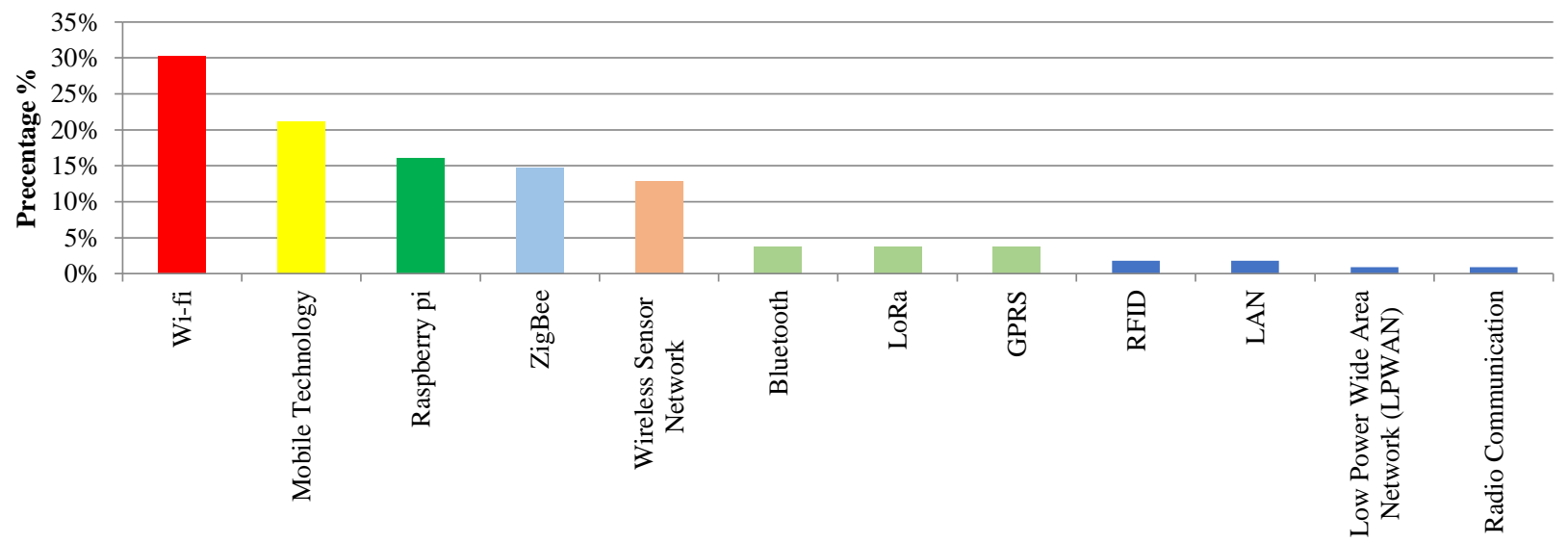

Technologies

Fig. 3. Overview of different Technologies Referred to in the Data Pool of 60 Peer Reviewed Published Articles and Frequency of Mentions Shown in Order of High Frequency to Low.

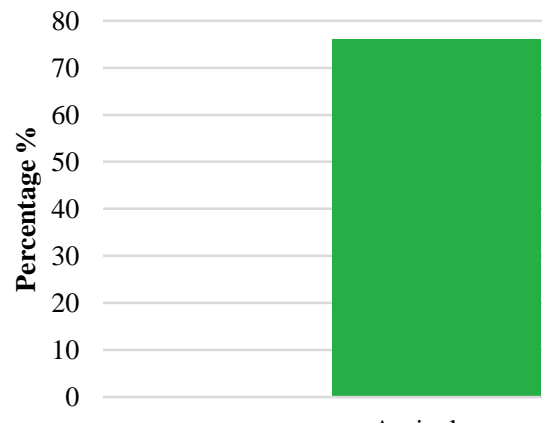

Agriculture

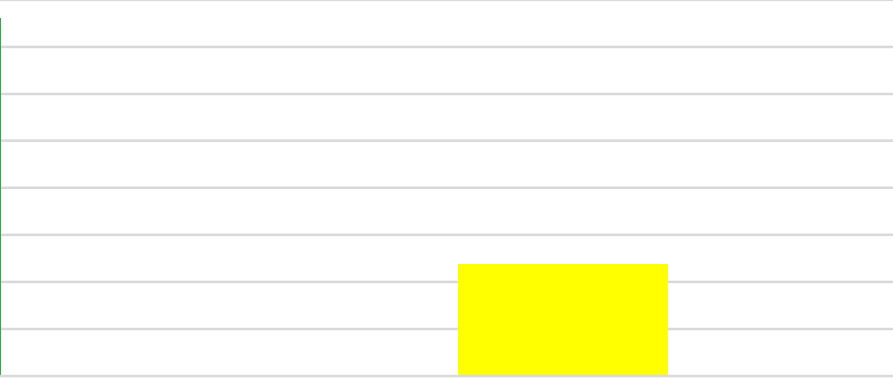

Farming

IoT Vertical

Fig. 4. Overview of Comparing the usage of Internet of Things in two Verticals as Agriculture and Farming in 60 Peer-Reviewed Research Articles to understand which is mostly used Internet of Things from Year 2016-2018. 
TABLE I. IOT IN AgRICULtURE AND FARMING CRITERION-APPROACH-DATA EXTRACTED FROM 60 SCIENTIFIC ARTICLES IN $2016-2018$

\begin{tabular}{|c|c|c|c|c|c|c|c|c|c|}
\hline $\begin{array}{l}\mathbf{N} \\
\mathbf{0}\end{array}$ & $\begin{array}{l}\text { Year/Autho } \\
\mathbf{r}\end{array}$ & $\begin{array}{l}\text { IoT Sub } \\
\text { Verticals }\end{array}$ & $\begin{array}{l}\text { Measures (Data } \\
\text { collection) }\end{array}$ & $\begin{array}{l}\text { Technologie } \\
\text { s Used }\end{array}$ & $\begin{array}{l}\text { Benefits of } \\
\text { Proposed } \\
\text { System }\end{array}$ & $\begin{array}{l}\text { Challenges in } \\
\text { Current } \\
\text { Approach }\end{array}$ & $\begin{array}{l}\text { Solution for } \\
\text { Current Issues }\end{array}$ & $\begin{array}{l}\text { Drivers of } \\
\text { IoT }\end{array}$ & $\begin{array}{l}\text { Applicatio } \\
\text { n }\end{array}$ \\
\hline 1 & $\begin{array}{l}\text { Venkate } \\
\text { et al } \\
(2017) \\
{[1]}\end{array}$ & $\begin{array}{l}\checkmark \text { Water } \\
\text { Managem } \\
\text { ent }\end{array}$ & $\begin{array}{l}\checkmark \text { Environmental } \\
\text { temperature } \\
\checkmark \text { Humidity } \\
\checkmark \text { Soil } \\
\checkmark \text { moisture }\end{array}$ & $\begin{array}{l}\checkmark \text { Raspberry } \\
\quad \text { pi } \\
\checkmark \text { Wi-Fi. } \\
\checkmark \text { RFID } \\
\checkmark \text { Bluetooth } \\
\checkmark \text { Zigbee }\end{array}$ & $\begin{array}{l}\checkmark \text { Can detect } \\
\text { the } \\
\text { temperature, } \\
\text { humidity } \\
\text { and } \\
\text { moisture. } \\
\checkmark \text { Continuous } \\
\text { monitoring } \\
\text { all the } \\
\text { places } \\
\text { including } \\
\text { critical } \\
\text { areas. }\end{array}$ & $\begin{array}{l}\checkmark \text { Human } \\
\text { interaction } \\
\checkmark \text { Labour cost } \\
\checkmark \text { Wastage of } \\
\text { water } \\
\checkmark \text { Crop from } \\
\text { abnormal } \\
\text { irrigation. }\end{array}$ & $\begin{array}{l}\checkmark \text { Detect } \\
\text { temperature, } \\
\text { humidity, } \\
\text { moisture using } \\
\text { sensors. } \\
\checkmark \text { Maximize the } \\
\text { yield of crop } \\
\text { by monitoring } \\
\text { agricultural } \\
\text { parameters. }\end{array}$ & $\begin{array}{l}\checkmark \text { Can deploy } \\
\text { it in any } \\
\text { type of } \\
\text { environmen } \\
t \text { for, } \\
\checkmark \text { monitoring } \\
\checkmark \text { flexibility } \\
\checkmark \text { robust }\end{array}$ & $\begin{array}{l}\checkmark \text { Agricultu } \\
\text { re }\end{array}$ \\
\hline 2 & $\begin{array}{l}\text { Athira et al } \\
(2017) \\
{[2]}\end{array}$ & $\begin{array}{l}\checkmark \text { Pest } \\
\text { controllin } \\
\mathrm{g} \\
\checkmark \text { Weather } \\
\quad \text { monitorin } \\
\mathrm{g}\end{array}$ & $\begin{array}{l}\checkmark \text { Soil moisture } \\
\checkmark \text { Temperature } \\
\checkmark \text { Water level }\end{array}$ & $\checkmark$ ZigBee & $\begin{array}{l}\checkmark \text { Irrigation } \\
\text { process is } \\
\text { completely } \\
\text { controlled } \\
\text { by } \\
\text { computer- } \\
\text { based } \\
\text { systems. } \\
\checkmark \text { System } \\
\text { analyses the } \\
\text { weather } \\
\text { reports. } \\
\checkmark \text { Keep pest } \\
\text { away from } \\
\text { the crops. } \\
\checkmark \text { Help to } \\
\text { faster the } \\
\text { growth of } \\
\text { plants. } \\
\checkmark \text { Power } \\
\text { efficient. }\end{array}$ & $\begin{array}{l}\checkmark \text { Only works } \\
\text { based on } \\
\text { the commands } \\
\text { from user }\end{array}$ & $\begin{array}{l}\checkmark \text { Low cost } \\
\checkmark \text { Efficient } \\
\text { growth of } \\
\text { crops } \\
\checkmark \text { Faster growth } \\
\text { of plants. }\end{array}$ & $\begin{array}{l}\checkmark \text { Predict and } \\
\text { tackle } \\
\text { drought } \\
\text { situations } \\
\text { to prevent } \\
\text { to loss of } \\
\text { crops. } \\
\checkmark \text { Keep } \\
\text { monitoring } \\
\text { climate } \\
\text { conditions. }\end{array}$ & $\begin{array}{l}\checkmark \text { Agricultu } \\
\text { re }\end{array}$ \\
\hline 3 & $\begin{array}{l}\text { Zhao et al } \\
(2017) \\
{[3]}\end{array}$ & $\begin{array}{l}\checkmark \text { Water } \\
\text { Managem } \\
\text { ent }\end{array}$ & $\checkmark$ Water level & $\begin{array}{l}\checkmark \text { LoRa } \\
\quad \text { technology }\end{array}$ & $\begin{array}{l}\checkmark \text { Can utilize } \\
\text { the water } \\
\text { usage. }\end{array}$ & $\begin{array}{l}\checkmark \text { To identify the } \\
\text { appropriate } \\
\text { time and in the } \\
\text { right amount } \\
\text { of water. } \\
\checkmark \text { High power } \\
\text { consumption. } \\
\checkmark \text { High cost. } \\
\checkmark \text { Low coverage } \\
\text { of ZigBee and } \\
\text { Wi-Fi. }\end{array}$ & $\begin{array}{l}\checkmark \text { Minimize the } \\
\text { cost of } \\
\text { deployment } \\
\text { and } \\
\text { maintenance. } \\
\checkmark \text { More efficient. } \\
\checkmark \text { Cover wider } \\
\text { area than } \\
\text { ZigBee and } \\
\text { Wi-Fi. } \\
\checkmark \text { Energy } \\
\text { consumption is } \\
\text { low. }\end{array}$ & $\begin{array}{l}\checkmark \text { Users can } \\
\text { remotely } \\
\text { access } \\
\text { irrigation } \\
\text { system and } \\
\text { check the } \\
\text { status. }\end{array}$ & $\begin{array}{l}\checkmark \text { Agricultu } \\
\quad \text { re }\end{array}$ \\
\hline 4 & $\begin{array}{l}\text { Sagar S et al } \\
(2017) \\
{[4]}\end{array}$ & $\begin{array}{l}\checkmark \text { Flood } \\
\text { Avoidance }\end{array}$ & $\begin{array}{l}\checkmark \text { Water level } \\
\checkmark \text { Soil moisture }\end{array}$ & $\begin{array}{l}\checkmark \text { Mobile } \\
\text { technology }\end{array}$ & $\begin{array}{l}\checkmark \text { Lessen the } \\
\text { human } \\
\text { intercession. } \\
\checkmark \text { Lessen the } \\
\text { probability } \\
\text { of the flood } \\
\text { occurrences. } \\
\checkmark \text { Faster the } \\
\text { growth of } \\
\text { the crops. }\end{array}$ & $\begin{array}{l}\checkmark \text { Save water for } \\
\text { the future. } \\
\checkmark \text { Save } \\
\text { electricity for } \\
\text { the future. }\end{array}$ & $\begin{array}{l}\checkmark \text { Flood } \\
\text { avoidance. } \\
\checkmark \text { Power cutoff is } \\
\text { being reduced. }\end{array}$ & $\begin{array}{l}\checkmark \text { Higher the } \\
\text { revenue by } \\
\text { faster the } \\
\text { growth of } \\
\text { crops. } \\
\checkmark \text { Ensure the } \\
\text { durability } \\
\text { of the soil. }\end{array}$ & $\begin{array}{l}\checkmark \text { Agricultu } \\
\text { re }\end{array}$ \\
\hline
\end{tabular}




\begin{tabular}{|c|c|c|c|c|c|c|c|c|c|}
\hline 5 & $\begin{array}{l}\text { Saraf et al } \\
(2017) \\
{[5]}\end{array}$ & $\begin{array}{l}\checkmark \text { Water } \\
\text { Managem } \\
\text { ent }\end{array}$ & $\begin{array}{l}\checkmark \text { Water level. } \\
\checkmark \text { Soil } \\
\checkmark \text { Moisture. } \\
\checkmark \text { Environment } \\
\text { temperature. } \\
\checkmark \text { Humidity }\end{array}$ & $\begin{array}{l}\checkmark \text { Wireless } \\
\text { sensor } \\
\text { network. } \\
\checkmark \text { ZigBee. } \\
\checkmark \text { Mobile } \\
\quad \text { technology } \\
.\end{array}$ & $\begin{array}{l}\checkmark \text { Lessen the } \\
\text { human } \\
\text { interaction. } \\
\checkmark \text { Efficiently } \\
\text { managed the } \\
\text { irrigation } \\
\text { water } \\
\text { system. }\end{array}$ & $\begin{array}{l}\checkmark \text { High water } \\
\text { consumption. } \\
\checkmark \text { Human } \\
\text { interaction. }\end{array}$ & $\begin{array}{l}\checkmark \text { The water } \\
\text { consumption is } \\
\text { reduced. } \\
\checkmark \text { Lessen the } \\
\text { human } \\
\text { interaction. }\end{array}$ & $\begin{array}{l}\checkmark \text { Reduced } \\
\text { water } \\
\text { consumptio } \\
\text { n. }\end{array}$ & $\begin{array}{l}\checkmark \text { Agricultu } \\
\text { re }\end{array}$ \\
\hline 6 & $\begin{array}{l}\text { Upadhyaya et } \\
\text { al (2017) } \\
{[6]}\end{array}$ & $\begin{array}{l}\checkmark \text { Water } \\
\text { Managem } \\
\text { ent }\end{array}$ & $\begin{array}{l}\checkmark \text { Soil Moisture } \\
\checkmark \text { Water } \\
\quad \text { requirements }\end{array}$ & $\begin{array}{l}\checkmark \text { Wi-Fi } \\
\checkmark \text { Mobile } \\
\quad \text { technology }\end{array}$ & $\begin{array}{l}\checkmark \text { Broader } \\
\text { coverage. } \\
\checkmark \text { Notify user } \\
\text { when any } \\
\text { change } \\
\text { happens. }\end{array}$ & $\begin{array}{l}\checkmark \text { No attention to } \\
\text { water } \\
\text { management. } \\
\checkmark \text { No economic } \\
\text { feasibility. } \\
\checkmark \text { Complicated } \\
\text { data for } \\
\text { understanding. } \\
\checkmark \text { Data display is } \\
\text { not user } \\
\text { friendly. }\end{array}$ & $\begin{array}{l}\checkmark \text { Water } \\
\text { requirements } \\
\text { monitored. } \\
\checkmark \text { Immediate } \\
\text { notification } \\
\text { sends to } \\
\text { farmer. } \\
\checkmark \text { User friendly } \\
\text { data collection. }\end{array}$ & $\begin{array}{l}\checkmark \text { Captured } \\
\text { moisture } \\
\text { values } \\
\text { stored in } \\
\text { the cloud. } \\
\checkmark \text { Compare } \\
\text { captured } \\
\text { values with } \\
\text { predefined } \\
\text { moisture } \\
\text { values. } \\
\checkmark \text { Used solar } \\
\text { powered } \\
\text { battery. }\end{array}$ & $\begin{array}{l}\checkmark \text { Agricultu } \\
\text { re }\end{array}$ \\
\hline 7 & $\begin{array}{l}\text { Udhayakuma } \\
\text { r S et al } \\
(2017) \\
{[7]}\end{array}$ & $\begin{array}{l}\checkmark \text { Water } \\
\text { Managem } \\
\text { ent }\end{array}$ & $\begin{array}{c}\checkmark \text { Soil moisture } \\
\checkmark \text { Environment } \\
\text { temperature. } \\
\checkmark \text { Humidity }\end{array}$ & $\begin{array}{l}\checkmark \text { Raspberry } \\
\text { pi } \\
\checkmark \text { Mobile } \\
\text { technology }\end{array}$ & $\begin{array}{l}\checkmark \text { Monitor } \\
\text { plants } \\
\text { through } \\
\text { smart } \\
\text { mobile. } \\
\checkmark \text { Efficient } \\
\text { water } \\
\text { supply } \\
\text { management } \\
.\end{array}$ & $\begin{array}{l}\checkmark \text { Overhead } \\
\text { sprinklers. } \\
\checkmark \text { Wastage of } \\
\text { water. }\end{array}$ & $\begin{array}{l}\checkmark \text { Watering crop } \\
\text { without human } \\
\text { interaction. }\end{array}$ & $\begin{array}{l}\checkmark \text { Analyze } \\
\text { moisture } \\
\text { level of } \\
\text { ground. }\end{array}$ & $\begin{array}{l}\checkmark \text { Agricultu } \\
\text { re }\end{array}$ \\
\hline 8 & $\begin{array}{l}\text { Kumar et al } \\
(2017) \\
{[8]}\end{array}$ & $\begin{array}{l}\checkmark \text { Water } \\
\text { Managem } \\
\text { ent }\end{array}$ & $\begin{array}{l}\checkmark \text { Soil moisture } \\
\checkmark \text { Environment } \\
\text { temperature. } \\
\checkmark \text { Humidity }\end{array}$ & $\begin{array}{l}\checkmark \text { Mobile } \\
\text { technology }\end{array}$ & $\begin{array}{l}\checkmark \text { Higher the } \\
\text { crop. } \\
\checkmark \text { Efficient } \\
\text { water } \\
\text { supply. } \\
\checkmark \text { Reduced } \\
\text { cost. } \\
\checkmark \text { Resource } \\
\text { optimization } \\
.\end{array}$ & $\begin{array}{l}\checkmark \text { Hard to water } \\
\text { to crop equally } \\
\text { due to unequal } \\
\text { rain water } \\
\text { distribution. } \\
\checkmark \text { Amount of } \\
\text { water not } \\
\text { defined. }\end{array}$ & $\begin{array}{l}\checkmark \text { Farmers can } \\
\text { know field } \\
\text { status even } \\
\text { they are at } \\
\text { home. } \\
\checkmark \text { Efficient water } \\
\text { management. } \\
\checkmark \text { Provide real } \\
\text { time } \\
\text { information. } \\
\end{array}$ & $\begin{array}{l}\checkmark \text { Automatic } \\
\text { plan } \\
\text { watering } \\
\text { system. }\end{array}$ & $\begin{array}{l}\checkmark \text { Agricultu } \\
\text { re }\end{array}$ \\
\hline 9 & $\begin{array}{l}\text { Mathew et al } \\
(2017) \\
{[9]}\end{array}$ & $\begin{array}{l}\checkmark \text { Nutrient } \\
\text { Managem } \\
\text { ent }\end{array}$ & $\begin{array}{l}\checkmark \text { Environment } \\
\text { temperature. } \\
\checkmark \text { Humidity } \\
\checkmark \text { Nitrogen level } \\
\checkmark \text { Prosperous level }\end{array}$ & $\begin{array}{l}\checkmark \text { Raspberry } \\
\text { pi } \\
\checkmark \text { Mobile } \\
\text { technology } \\
\checkmark \text { Wi-Fi }\end{array}$ & $\begin{array}{l}\checkmark \text { Can monitor } \\
\text { whether } \\
\text { conditions. } \\
\checkmark \text { Cost } \\
\text { effective } \\
\checkmark \text { Automatical } \\
\text { ly } \\
\text { monitored } \\
\text { disease } \\
\text { associated } \\
\text { with rice } \\
\text { species. } \\
\end{array}$ & $\begin{array}{l}\checkmark \text { Low or high } \\
\text { watering. } \\
\checkmark \text { Lack of } \\
\text { nutrition } \\
\text { management. }\end{array}$ & $\begin{array}{l}\checkmark \text { Whether } \\
\text { conditions } \\
\text { detected. } \\
\checkmark \text { Enhanced the } \\
\text { fertilizer } \\
\text { amount. }\end{array}$ & $\begin{array}{l}\checkmark \text { Can } \\
\text { enhance the } \\
\text { fertilizer } \\
\text { amount. }\end{array}$ & $\begin{array}{l}\checkmark \text { Agricultu } \\
\text { re }\end{array}$ \\
\hline 10 & $\begin{array}{l}\text { Suhas et al } \\
(2017) \\
{[10]}\end{array}$ & $\begin{array}{l}\checkmark \text { Water } \\
\text { Managem } \\
\text { ent }\end{array}$ & $\begin{array}{l}\checkmark \text { Temperature } \\
\checkmark \text { Moisture level } \\
\checkmark \text { Humidity } \\
\checkmark \text { Light Intensity } \\
\checkmark \text { Nitrogen, } \\
\checkmark \text { Phosphorus } \\
\checkmark \text { Potassium }\end{array}$ & $\begin{array}{l}\checkmark \text { Bluetooth } \\
\checkmark \text { Wi-Fi }\end{array}$ & $\begin{array}{l}\checkmark \text { Cost } \\
\text { effective. } \\
\checkmark \text { High } \\
\text { efficient } \\
\text { water } \\
\text { management } \\
.\end{array}$ & $\begin{array}{c}\checkmark \text { Higher water } \\
\text { consumption. } \\
\checkmark \text { High power } \\
\text { utilization. } \\
\checkmark \text { Lack of useful } \\
\text { inference. }\end{array}$ & $\begin{array}{l}\checkmark \text { Reduced water } \\
\text { consumption. } \\
\checkmark \text { Better power } \\
\text { utilization. }\end{array}$ & $\begin{array}{l}\checkmark \text { Automated } \\
\text { water } \\
\text { supply } \\
\text { system. }\end{array}$ & $\begin{array}{l}\checkmark \text { Agricultu } \\
\text { re }\end{array}$ \\
\hline
\end{tabular}




\begin{tabular}{|c|c|c|c|c|c|c|c|c|c|}
\hline 11 & $\begin{array}{l}\text { Wicha et al } \\
(2017) \\
{[11]}\end{array}$ & $\begin{array}{l}\checkmark \text { Water } \\
\text { Managem } \\
\text { ent }\end{array}$ & $\begin{array}{l}\checkmark \text { Soil level } \\
\checkmark \text { Temperature }\end{array}$ & $\checkmark \mathrm{Wi}-\mathrm{Fi}$ & $\begin{array}{l}\checkmark \text { Efficient } \\
\text { water } \\
\text { management } \\
.\end{array}$ & $\begin{array}{l}\checkmark \text { High water } \\
\text { consumption. }\end{array}$ & $\begin{array}{l}\checkmark \text { Managed water } \\
\text { system } \\
\text { effective } \\
\text { manner. }\end{array}$ & $\begin{array}{l}\checkmark \text { Reveals the } \\
\text { positive } \\
\text { comparison } \\
\text { results } \\
\text { from the } \\
\text { adaptive } \\
\text { Wetting } \\
\text { Front } \\
\text { Detector } \\
\text { (WFD). }\end{array}$ & $\begin{array}{l}\checkmark \text { Agricultu } \\
\text { re }\end{array}$ \\
\hline 12 & $\begin{array}{l}\text { Rajakumar et } \\
\text { al (2017) } \\
{[12]}\end{array}$ & $\begin{array}{l}\checkmark \text { Crop } \\
\text { production }\end{array}$ & $\begin{array}{l}\checkmark \text { Soil level } \\
\checkmark \text { Soil nutrient }\end{array}$ & $\begin{array}{l}\checkmark \text { Mobile } \\
\text { technology }\end{array}$ & $\begin{array}{l}\checkmark \text { Increase the } \\
\text { crop } \\
\text { production. } \\
\checkmark \text { Can get } \\
\text { current } \\
\text { fertilizer } \\
\text { requirement } \\
\text { s. }\end{array}$ & $\begin{array}{l}\checkmark \text { Due to } \\
\text { improper } \\
\text { maintenance, } \\
\text { the crop } \\
\text { becomes } \\
\text { damaged } \\
\text { which causes a } \\
\text { huge loss for a } \\
\text { farmer. }\end{array}$ & $\begin{array}{l}\checkmark \text { Enhance the } \\
\text { crop. } \\
\checkmark \text { Control the } \\
\text { agricultural } \\
\text { product costs. }\end{array}$ & $\begin{array}{l}\checkmark \text { Interfacing } \\
\text { different } \\
\text { soil } \\
\text { nutrient } \\
\text { sensors. }\end{array}$ & $\begin{array}{l}\checkmark \text { Agricultu } \\
\text { re }\end{array}$ \\
\hline 13 & $\begin{array}{l}\text { Sachapara et } \\
\text { al (2017) } \\
{[13]}\end{array}$ & $\begin{array}{l}\checkmark \text { Crop } \\
\text { Productio } \\
\mathrm{n} \\
\checkmark \text { Water } \\
\quad \text { Managem } \\
\text { ent }\end{array}$ & $\begin{array}{l}\checkmark \text { Temperature } \\
\checkmark \text { Humidity } \\
\checkmark \text { Soil } \\
\checkmark \text { moisture } \\
\checkmark \text { Leaf wetness } \\
\checkmark \text { Wind } \\
\text { speed/direction } \\
\checkmark \text { Rainfall } \\
\text { detection } \\
\checkmark \text { Soil ph. } \\
\checkmark \text { Seed } \\
\text { recognition. }\end{array}$ & $\begin{array}{l}\checkmark \text { Raspberry } \\
\text { pi } \\
\checkmark \text { Mobile } \\
\text { technology }\end{array}$ & $\begin{array}{l}\checkmark \text { Enhanced } \\
\text { crop } \\
\text { production. } \\
\checkmark \text { Enhanced } \\
\\
\quad \text { quality. } \\
\checkmark \text { Reduced } \\
\text { costs. }\end{array}$ & $\begin{array}{l}\checkmark \text { Poor risk } \\
\text { management. } \\
\checkmark \text { Poor water } \\
\text { management. } \\
\checkmark \text { Poor } \\
\text { infrastructure. } \\
\checkmark \text { Poor crops } \\
\text { yield and big } \\
\text { loss for } \\
\text { farmers. }\end{array}$ & $\begin{array}{l}\checkmark \text { Enhanced } \\
\text { crops yield by } \\
\text { proper water } \\
\text { management. }\end{array}$ & $\begin{array}{l}\checkmark \text { Seed } \\
\text { recognition } \\
\text { system } \\
\text { helps to } \\
\text { know } \\
\text { sustainable } \\
\text { environmen } \\
\text { tal } \\
\text { conditions. }\end{array}$ & $\begin{array}{l}\checkmark \text { Agricultu } \\
\text { re }\end{array}$ \\
\hline 14 & $\begin{array}{l}\text { Pooja S et al } \\
(2017) \\
{[14]}\end{array}$ & $\begin{array}{l}\checkmark \text { Weather } \\
\text { Monitorin } \\
\mathrm{g} \\
\checkmark \text { Precision } \\
\text { Farming }\end{array}$ & $\begin{array}{l}\checkmark \text { Temperature } \\
\checkmark \text { Humidity } \\
\checkmark \text { Soil } \\
\checkmark \text { Moisture } \\
\checkmark \text { Light intensity }\end{array}$ & $\begin{array}{l}\checkmark \text { Raspberry- } \\
\quad \mathrm{Pi} \\
\checkmark \mathrm{Wi}-\mathrm{Fi}\end{array}$ & $\begin{array}{l}\checkmark \text { Improve the } \\
\text { crop } \\
\text { traceability. } \\
\checkmark \text { Increase } \\
\text { overall } \\
\text { yield. }\end{array}$ & $\begin{aligned} & \checkmark \text { Wastage of } \\
& \text { crops. } \\
& \checkmark \text { Poor water } \\
& \text { system } \\
& \text { management. }\end{aligned}$ & $\begin{array}{l}\checkmark \text { Crop } \\
\text { productivity } \\
\text { increased. } \\
\checkmark \text { Reduced } \\
\text { wastage of } \\
\text { crops. } \\
\checkmark \text { Reduced water } \\
\text { use. } \\
\checkmark \text { Minimal } \\
\text { maintenance } \\
\text { required. } \\
\checkmark \text { High accuracy }\end{array}$ & $\begin{array}{l}\checkmark \text { Use of } \\
\text { decision } \\
\text { making } \\
\text { algorithm. }\end{array}$ & $\begin{array}{l}\checkmark \text { Agricultu } \\
\text { re }\end{array}$ \\
\hline 15 & $\begin{array}{l}\text { Kavitha et al } \\
(2017) \\
{[15]}\end{array}$ & $\begin{array}{l}\checkmark \text { Crop } \\
\text { manageme } \\
\text { nt }\end{array}$ & $\begin{array}{l}\checkmark \text { Soil moisture } \\
\checkmark \text { pH level } \\
\checkmark \text { Temperature } \\
\checkmark \text { Humidity } \\
\checkmark \text { Light intensity } \\
\checkmark \text { Water level }\end{array}$ & $\begin{array}{l}\checkmark \text { Mobile } \\
\text { technology }\end{array}$ & $\begin{array}{l}\checkmark \text { Improved } \\
\text { crop growth. } \\
\checkmark \text { Efficient } \\
\text { watering } \\
\text { system. }\end{array}$ & $\begin{array}{l}\checkmark \text { Difficulties in } \\
\text { monitoring. } \\
\checkmark \text { Harvesting } \\
\text { related } \\
\text { problems. } \\
\checkmark \text { Poor crop } \\
\text { growth. } \\
\checkmark \text { Poor power } \\
\text { management. } \\
\checkmark \text { Poor water } \\
\text { management. }\end{array}$ & $\begin{aligned} & \checkmark \text { Effective water } \\
& \text { management. } \\
& \checkmark \text { Effective } \\
& \text { power } \\
& \text { management. }\end{aligned}$ & $\begin{array}{l}\checkmark \text { Reduced } \\
\text { costs } \\
\text { between } \\
\text { central } \\
\text { server and } \\
\text { software. }\end{array}$ & $\begin{array}{l}\checkmark \text { Agricultu } \\
\text { re }\end{array}$ \\
\hline 16 & $\begin{array}{l}\text { Jawahar et al } \\
(2017) \\
{[16]}\end{array}$ & $\begin{array}{l}\checkmark \text { Crop } \\
\text { manageme } \\
\text { nt } \\
\checkmark \text { Water } \\
\text { Managem } \\
\text { ent }\end{array}$ & $\begin{array}{l}\checkmark \text { Temperature } \\
\checkmark \text { Humidity } \\
\checkmark \text { Soil moisture } \\
\checkmark \text { Water level }\end{array}$ & $\begin{array}{l}\checkmark \text { Mobile } \\
\text { technology }\end{array}$ & $\begin{array}{l}\checkmark \text { Prevent } \\
\quad \text { crops from } \\
\text { spoilage } \\
\text { during rain. } \\
\checkmark \text { Recycling } \\
\text { rain water in } \\
\text { an efficient } \\
\text { manner. }\end{array}$ & $\begin{array}{l}\checkmark \text { Wastage of } \\
\text { water. } \\
\checkmark \text { Human } \\
\text { interaction. } \\
\checkmark \text { Hard to } \\
\text { monitor field } \\
\text { every time to } \\
\text { avoid } \\
\text { intrusion } \\
\text { attacks. } \\
\end{array}$ & $\begin{array}{l}\checkmark \text { Update farmer } \\
\text { with live } \\
\text { condition of } \\
\text { the field. } \\
\checkmark \text { Lessen human } \\
\text { interaction. } \\
\checkmark \text { Notify } \\
\text { intrusion } \\
\text { detections with } \\
\text { an alarm. } \\
\end{array}$ & $\begin{array}{l}\checkmark \text { Excess } \\
\text { water from } \\
\text { the } \\
\text { cultivation } \\
\text { field and } \\
\text { recycled } \\
\text { back to the } \\
\text { tank. }\end{array}$ & $\begin{array}{l}\checkmark \text { Agricultu } \\
\text { re }\end{array}$ \\
\hline
\end{tabular}




\begin{tabular}{|c|c|c|c|c|c|c|c|c|c|}
\hline 17 & $\begin{array}{l}\text { Nibi K V et } \\
\text { al (2017) } \\
{[17]}\end{array}$ & $\begin{array}{l}\checkmark \text { Whether } \\
\text { manageme } \\
\text { nt } \\
\checkmark \text { Crop } \\
\text { manageme } \\
\text { nt }\end{array}$ & $\begin{array}{l}\checkmark \text { Soil moisture } \\
\checkmark \text { Temperature } \\
\checkmark \text { pH level }\end{array}$ & $\begin{array}{l}\checkmark \text { Mobile } \\
\text { technology } \\
\checkmark \mathrm{Wi-Fi}\end{array}$ & $\begin{array}{l}\checkmark \text { Provide } \\
\text { advice for } \\
\text { farmers to } \\
\text { properly } \\
\text { grow and } \\
\text { treat the } \\
\text { crops. } \\
\checkmark \text { Provide } \\
\text { suggestions } \\
\text { to } \\
\text { monitoring } \\
\text { crops. } \\
\checkmark \text { Ex: } \\
\text { Irrigation } \\
\text { timings } \\
\checkmark \text { Optimum } \\
\text { usage of } \\
\text { fertilizers. } \\
\checkmark \text { Provide } \\
\text { whether } \\
\text { information. }\end{array}$ & $\begin{array}{l}\checkmark \text { High human } \\
\text { interaction. } \\
\checkmark \text { Hard to deal } \\
\text { with changing } \\
\text { whether } \\
\text { parameters. }\end{array}$ & $\begin{array}{l}\checkmark \text { Provide } \\
\text { efficient } \\
\text { suggestions } \\
\text { about when } \\
\text { and how mush } \\
\text { to irrigate. } \\
\checkmark \text { Provide } \\
\text { adequate } \\
\text { fertilizer } \\
\text { information. }\end{array}$ & $\begin{array}{l}\checkmark \text { Provide } \\
\text { farmer } \\
\text { friendly } \\
\text { alerts and } \\
\text { guidance } \\
\text { with their } \\
\text { local } \\
\text { language. }\end{array}$ & $\begin{array}{l}\checkmark \text { Agricultu } \\
\text { re }\end{array}$ \\
\hline 18 & $\begin{array}{l}\text { Tran et al } \\
(2017) \\
{[18]}\end{array}$ & $\begin{array}{l}\checkmark \text { Crop } \\
\text { manageme } \\
\text { nt } \\
\checkmark \text { Nutrient } \\
\text { Detection }\end{array}$ & $\begin{array}{l}\checkmark \text { Temperature } \\
\checkmark \text { Humidity }\end{array}$ & $\begin{array}{l}\checkmark \text { ZigBee } \\
\checkmark \text { Raspberry } \\
\quad \mathrm{Pi}\end{array}$ & $\begin{array}{l}\checkmark \text { Could } \\
\text { prevent soil } \\
\text { erosion. }\end{array}$ & $\begin{array}{l}\checkmark \text { High energy } \\
\text { consumption. } \\
\checkmark \text { Soil and } \\
\text { nutrient } \\
\text { depletion. }\end{array}$ & $\begin{array}{l}\checkmark \text { Reduced } \\
\text { energy } \\
\text { consumption. } \\
\checkmark \text { Could react } \\
\text { changes in } \\
\text { environment } \\
\text { and soil. }\end{array}$ & $\begin{array}{l}\checkmark \text { Reduce the } \\
\text { consumptio } \\
n \text { of energy } \\
\checkmark \text { Increase } \\
\text { the number } \\
\text { of sensors }\end{array}$ & $\begin{array}{l}\checkmark \text { Agricultu } \\
\text { re }\end{array}$ \\
\hline 19 & $\begin{array}{l}\text { Muhammad } \\
\text { et al (2017) } \\
{[19]}\end{array}$ & $\begin{array}{l}\checkmark \text { Water } \\
\text { Managem } \\
\text { ent }\end{array}$ & $\begin{array}{l}\checkmark \text { Water level } \\
\checkmark \text { Soil Moisture }\end{array}$ & $\begin{array}{l}\text { Wireless } \\
\quad \text { Sensor } \\
\text { Network } \\
\checkmark \text { Radio } \\
\text { Communica } \\
\text { tion }\end{array}$ & $\begin{array}{l}\checkmark \text { Efficient } \\
\text { water } \\
\text { management } \\
\end{array}$ & $\begin{array}{l}\checkmark \text { Climate } \\
\text { changes. } \\
\checkmark \text { Scarcity of } \\
\text { water. }\end{array}$ & $\begin{array}{l}\checkmark \text { Monitoring } \\
\text { water in } \\
\text { watercourses. }\end{array}$ & $\begin{array}{l}\checkmark \text { Smart } \\
\text { water } \\
\text { metering } \\
\text { system. }\end{array}$ & $\begin{array}{l}\checkmark \text { Agricultu } \\
\text { re }\end{array}$ \\
\hline 20 & $\begin{array}{l}\text { Viswanathan } \\
\text { et al (2017) } \\
{[20]}\end{array}$ & $\begin{array}{l}\checkmark \text { Crop } \\
\text { manageme } \\
\text { nt. } \\
\checkmark \text { Warehous } \\
\text { e } \\
\quad \text { Managem } \\
\text { ent. }\end{array}$ & $\begin{array}{l}\checkmark \text { Temperature } \\
\checkmark \text { Humidity } \\
\checkmark \text { Soil Moisture } \\
\checkmark \text { Rain fall } \\
\checkmark \text { Light intensity }\end{array}$ & $\checkmark \mathrm{Wi}-\mathrm{Fi}$ & $\begin{array}{l}\checkmark \text { Remote } \\
\text { controlled } \\
\text { processes to } \\
\text { perform } \\
\text { such tasks } \\
\text { as; } \\
\checkmark \text { Spraying } \\
\checkmark \text { Weeding } \\
\checkmark \text { Bird and } \\
\text { animal } \\
\text { scaring } \\
\checkmark \text { Keeping } \\
\text { vigilance } \\
\checkmark \text { Provide } \\
\text { smart } \\
\text { warehouse } \\
\text { management } \\
\checkmark \\
\checkmark \text { Theft } \\
\text { detection in } \\
\text { warehouse. }\end{array}$ & $\begin{array}{l}\checkmark \text { High cost } \\
\checkmark \text { Human } \\
\text { interaction for } \\
\text { all activities. }\end{array}$ & $\begin{array}{l}\checkmark \text { Reduced cost. } \\
\checkmark \text { Lessen human } \\
\text { interaction. } \\
\checkmark \text { High } \\
\text { reliability. } \\
\checkmark \text { Improved crop } \\
\text { production. }\end{array}$ & $\begin{array}{l}\checkmark \text { Smart } \\
\text { warehouse } \\
\text { manageme } \\
\text { nt. }\end{array}$ & $\begin{array}{l}\checkmark \text { Agricultu } \\
\text { re }\end{array}$ \\
\hline 21 & $\begin{array}{l}\text { Dai et al } \\
(2017) \\
{[21]}\end{array}$ & $\begin{array}{l}\checkmark \text { Water } \\
\text { Managem } \\
\text { ent } \\
\checkmark \text { Agricultur } \\
\text { al } \\
\text { Greenhous } \\
\text { e } \\
\text { Managem } \\
\text { ent }\end{array}$ & $\begin{array}{l}\checkmark \text { Soli } \\
\quad \text { environment: } \\
\checkmark \text { Temperature } \\
\checkmark \text { Humidity of soil } \\
\checkmark \text { Soil CO2 } \\
\checkmark \text { Soil pH } \\
\checkmark \text { Environmental: } \\
\checkmark \text { Temperature } \\
\checkmark \text { Humidity } \\
\checkmark \text { Wind speed } \\
\checkmark \text { Air pressure } \\
\checkmark \text { Rainfall }\end{array}$ & $\checkmark$ ZigBee & $\begin{array}{l}\checkmark \text { High } \\
\quad \text { irrigation } \\
\text { efficiency. } \\
\checkmark \text { High } \\
\quad \text { flexibility. }\end{array}$ & $\begin{array}{l}\checkmark \text { Low irrigation } \\
\text { efficiency } \\
\checkmark \text { High labour } \\
\text { cost } \\
\checkmark \text { Low precision } \\
\checkmark \text { High water } \\
\text { consumption. }\end{array}$ & $\begin{array}{l}\checkmark \text { Lessen labour } \\
\text { cost. } \\
\checkmark \text { Reduced water } \\
\text { wastage. }\end{array}$ & $\begin{array}{l}\checkmark \text { Powerful } \\
\text { servers to } \\
\text { handle } \\
\text { storage } \\
\text { data. }\end{array}$ & $\begin{array}{l}\checkmark \text { Agricultu } \\
\text { re }\end{array}$ \\
\hline 22 & $\begin{array}{l}\text { Yuan et al } \\
(2017) \\
{[22]}\end{array}$ & $\begin{array}{l}\checkmark \text { Agricultur } \\
\text { al } \\
\text { Greenhous } \\
\text { e } \\
\text { Managem } \\
\text { ent }\end{array}$ & $\begin{array}{l}\checkmark \text { Temperature } \\
\checkmark \text { Humidity }\end{array}$ & $\checkmark$ ZigBee & $\begin{array}{l}\checkmark \text { More } \\
\text { flexible. } \\
\checkmark \text { Low power } \\
\text { consuming. }\end{array}$ & $\begin{aligned} & \checkmark \text { Short distance } \\
& \text { communicatio } \\
& \mathrm{n} . \\
& \checkmark \text { High power } \\
& \text { consumption. }\end{aligned}$ & $\begin{array}{l}\checkmark \text { Lessen power } \\
\text { consumption. }\end{array}$ & $\begin{array}{l}\checkmark \text { Automated } \\
\text { greenhouse } \\
\text { manageme } \\
\text { nt. }\end{array}$ & $\begin{array}{l}\checkmark \text { Agricultu } \\
\text { re }\end{array}$ \\
\hline
\end{tabular}




\begin{tabular}{|c|c|c|c|c|c|c|c|c|c|}
\hline 23 & $\begin{array}{l}\text { Garcia et al } \\
(2017) \\
{[23]}\end{array}$ & $\begin{array}{l}\checkmark \text { Water } \\
\text { Managem } \\
\text { ent } \\
\checkmark \text { Energy } \\
\text { Managem } \\
\text { ent }\end{array}$ & $\begin{array}{l}\checkmark \text { Irrigation evets } \\
\quad \text { as: } \\
\checkmark \text { Flow level } \\
\checkmark \text { Pressure level } \\
\checkmark \text { Wind speed }\end{array}$ & $\begin{array}{l}\checkmark \text { LoRa } \\
\checkmark \text { Wi-Fi }\end{array}$ & $\begin{array}{l}\checkmark \text { Low cost } \\
\text { irrigation } \\
\text { control. } \\
\checkmark \text { Autonomou } \\
\text { s decision } \\
\text { making } \\
\text { without } \\
\text { human } \\
\text { interactions. }\end{array}$ & $\begin{array}{l}\checkmark \text { Device } \\
\text { scalability is } \\
\text { low. } \\
\checkmark \text { Device } \\
\text { manageability } \\
\text { is low. }\end{array}$ & $\begin{array}{l}\checkmark \text { Lessen human } \\
\text { interaction. } \\
\checkmark \text { Efficient water } \\
\text { management. } \\
\checkmark \text { Efficient } \\
\text { power } \\
\text { management. }\end{array}$ & $\begin{array}{l}\checkmark \text { Autonomou } \\
\text { s decision } \\
\text { making } \\
\text { without } \\
\text { human } \\
\text { interactions } \\
.\end{array}$ & $\begin{array}{l}\checkmark \text { Agricultu } \\
\text { re }\end{array}$ \\
\hline 24 & $\begin{array}{l}\text { Janani V et al } \\
(2017) \\
{[24]}\end{array}$ & $\begin{array}{l}\checkmark \text { Soil } \\
\text { Managem } \\
\text { ent } \\
\checkmark \text { Nutrient } \\
\text { Detection }\end{array}$ & $\begin{array}{l}\checkmark \text { Soil Measures: } \\
\checkmark \text { Soil pH } \\
\checkmark \text { Soil } \\
\text { Temperature } \\
\checkmark \text { Soil Humidity }\end{array}$ & $\begin{array}{l}\checkmark \text { Wi-Fi } \\
\checkmark \text { Raspberry } \\
\quad \mathrm{Pi}\end{array}$ & $\begin{array}{l}\checkmark \text { Reduced } \\
\text { manual } \\
\text { monitoring } \\
\text { of the field. } \\
\checkmark \\
\checkmark \text { Obtained } \\
\text { nature of } \\
\text { soil. } \\
\checkmark \text { Can } \\
\text { monitored } \\
\text { from } \\
\text { anywhere. }\end{array}$ & $\begin{array}{l}\checkmark \text { Manual field } \\
\text { monitoring. } \\
\checkmark \text { Cost is high. } \\
\checkmark \text { Difficult to } \\
\text { predict the } \\
\text { crop for the } \\
\text { field. }\end{array}$ & $\begin{array}{l}\checkmark \text { Reduces the } \\
\text { difficulty for } \\
\text { identify the } \\
\text { right crop for } \\
\text { the field. } \\
\checkmark \text { Increased } \\
\text { agricultural } \\
\text { production } \\
\checkmark \text { Reduced time } \\
\text { and money for } \\
\text { farmers. }\end{array}$ & $\begin{array}{l}\checkmark \text { Soli } \\
\text { manageme } \\
\text { nt with the } \\
\text { attention of } \\
\text { nutrient, } \\
\text { fertilization } \\
.\end{array}$ & $\begin{array}{l}\checkmark \text { Agricultu } \\
\text { re }\end{array}$ \\
\hline 25 & $\begin{array}{l}\text { Jyothi et al } \\
(2017) \\
{[25]}\end{array}$ & $\begin{array}{l}\checkmark \text { Crop } \\
\text { Managem } \\
\text { ent }\end{array}$ & $\begin{array}{l}\checkmark \text { Temperature } \\
\checkmark \text { Humidity }\end{array}$ & $\begin{array}{l}\checkmark \text { Wi-Fi } \\
\checkmark \text { GPRS }\end{array}$ & $\begin{array}{l}\checkmark \text { Provide } \\
\text { accurate } \\
\text { changes in } \\
\text { the crop } \\
\text { yield. } \\
\checkmark \text { Advance the } \\
\text { harvest of } \\
\text { the crop. }\end{array}$ & $\begin{array}{l}\checkmark \text { Reduced } \\
\text { human power. } \\
\checkmark \text { High cost. } \\
\checkmark \text { High power } \\
\quad \text { consumption. }\end{array}$ & $\begin{array}{l}\checkmark \text { Automated } \\
\text { monitoring of } \\
\text { the crop. } \\
\checkmark \text { Notify } \\
\text { corrective } \\
\text { actions to be } \\
\text { taken. } \\
\checkmark \text { Low cost. } \\
\checkmark \text { Consume less } \\
\text { Power. }\end{array}$ & $\begin{array}{l}\checkmark \text { Notify } \\
\text { agricultural } \\
\text { fields with } \\
\text { a MMS to } \\
\text { the farer. }\end{array}$ & $\begin{array}{l}\checkmark \text { Agricultu } \\
\text { re }\end{array}$ \\
\hline 26 & $\begin{array}{l}\text { Javale et al } \\
(2017) \\
{[26]}\end{array}$ & $\begin{array}{l}\checkmark \text { Water } \\
\text { Managem } \\
\text { ent }\end{array}$ & $\begin{array}{l}\checkmark \text { Soil moisture } \\
\checkmark \text { Soli temperature } \\
\checkmark \text { Soil pH } \\
\checkmark \text { Soil water level }\end{array}$ & $\begin{array}{l}\checkmark \text { Mobile } \\
\text { technology }\end{array}$ & $\begin{array}{l}\checkmark \text { Help to } \\
\text { irrigate } \\
\text { farms } \\
\text { efficiently. } \\
\checkmark \text { Lessen } \\
\quad \text { human } \\
\text { interaction. }\end{array}$ & $\begin{array}{l}\checkmark \text { Water scarcity } \\
\checkmark \text { Human } \\
\quad \text { interaction is } \\
\text { high. }\end{array}$ & $\begin{array}{l}\checkmark \text { Estimates } \\
\text { water as per } \\
\text { requirements. } \\
\checkmark \text { Depending on } \\
\text { soli and crop, } \\
\text { fertilizer } \\
\text { suggestions } \\
\text { provided. } \\
\checkmark \text { Estimate the } \\
\text { rainfall based } \\
\text { on whether } \\
\text { forecast. } \\
\checkmark \text { Manage water } \\
\text { level according } \\
\text { to the predicted } \\
\text { rainfall. }\end{array}$ & $\begin{array}{l}\checkmark \text { Water } \\
\text { supply can } \\
\text { control by } \\
\text { mobile } \\
\text { application } \\
\text { with the } \\
\text { less human } \\
\text { interaction. }\end{array}$ & $\begin{array}{l}\checkmark \text { Agricultu } \\
\text { re }\end{array}$ \\
\hline 27 & $\begin{array}{l}\text { Sathyadevan } \\
\text { et al (2017) } \\
{[27]}\end{array}$ & $\begin{array}{l}\checkmark \text { Soil } \\
\text { Quality } \\
\text { Managem } \\
\text { ent } \\
\checkmark \text { Water } \\
\quad \text { Managem } \\
\text { ent }\end{array}$ & $\begin{array}{l}\checkmark \text { Temperature } \\
\checkmark \text { Humidity } \\
\checkmark \text { Soil moisture } \\
\checkmark \text { Water level }\end{array}$ & $\begin{array}{l}\checkmark \text { ZigBee } \\
\checkmark \text { Wi-Fi } \\
\checkmark \text { Mobile } \\
\text { technology }\end{array}$ & $\begin{array}{l}\checkmark \text { Accurate } \\
\text { measuremen } \\
\text { ts of the } \\
\text { velocity of } \\
\text { the liquid } \\
\text { flowing } \\
\text { inside the } \\
\text { pipe. } \\
\checkmark \text { Pump } \\
\text { monitoring. }\end{array}$ & $\begin{array}{l}\checkmark \text { Water } \\
\text { conservation. } \\
\checkmark \text { High labour } \\
\text { cost. } \\
\checkmark \text { High } \\
\text { electricity } \\
\text { consumption. } \\
\checkmark \text { Overdependen } \\
\text { ce on the } \\
\text { chemical } \\
\text { fertilizers. }\end{array}$ & $\begin{array}{l}\checkmark \text { Automated } \\
\text { water } \\
\text { management. } \\
\checkmark \text { Lessen labour } \\
\text { cost. } \\
\checkmark \text { Lessen power } \\
\text { usage. }\end{array}$ & $\begin{array}{l}\checkmark \text { Multiple } \\
\text { sensor data } \\
\text { collected } \\
\text { into IoT } \\
\text { framework. }\end{array}$ & $\begin{array}{l}\checkmark \text { Agricultu } \\
\text { re }\end{array}$ \\
\hline 28 & $\begin{array}{l}\text { Kulkarni et al } \\
(2017) \\
{[28]}\end{array}$ & $\begin{aligned} & \checkmark \text { Soil } \\
& \text { manageme } \\
& \text { nt } \\
& \checkmark \text { Water } \\
& \text { Managem } \\
& \text { ent }\end{aligned}$ & $\begin{array}{l}\checkmark \text { Soil moisture } \\
\checkmark \text { Soil pH }\end{array}$ & $\begin{array}{l}\checkmark \text { Android } \\
\checkmark \text { Wi-Fi }\end{array}$ & $\begin{array}{l}\checkmark \text { Decreased } \\
\text { cost in } \\
\text { manufacturi } \\
\text { ng. } \\
\checkmark \text { Reduced } \\
\text { cost in } \\
\text { maintenance } \\
\quad .\end{array}$ & $\begin{aligned} & \checkmark \text { High chemical } \\
& \text { fertilizer use. } \\
& \checkmark \text { High energy } \\
& \text { consumption. } \\
& \checkmark \text { High fertilizer } \\
& \text { costs. }\end{aligned}$ & $\begin{aligned} & \checkmark \text { Save water } \\
& \text { more } \\
& \text { efficiently. } \\
& \checkmark \text { Increase the } \\
& \text { crop yield. } \\
& \checkmark \text { Save energy } \\
& \text { costs. } \\
& \checkmark \text { Save fertilizer } \\
& \text { costs. }\end{aligned}$ & $\begin{array}{l}\checkmark \text { Monitor } \\
\text { and control } \\
\text { plant } \\
\text { growth } \\
\text { parameters. } \\
\checkmark \text { Replaces } \\
\text { the current } \\
\text { system for } \\
\text { soil } \\
\text { moisture, } \\
\text { pH and } \\
\text { salinity } \\
\text { value } \\
\text { testing. }\end{array}$ & $\begin{array}{l}\checkmark \text { Agricultu } \\
\text { re }\end{array}$ \\
\hline 29 & $\begin{array}{l}\text { Mahalakshmi } \\
\text { et al (2016) } \\
{[29]}\end{array}$ & $\begin{array}{l}\checkmark \text { Water } \\
\text { Managem } \\
\text { ent }\end{array}$ & $\begin{array}{l}\checkmark \text { Temperature } \\
\checkmark \text { Humidity } \\
\checkmark \text { Soil Moisture }\end{array}$ & $\checkmark$ ZigBee & $\begin{array}{l}\checkmark \text { Monitor } \\
\text { crop field. } \\
\checkmark \text { Automate }\end{array}$ & $\begin{array}{l}\checkmark \text { Water } \\
\text { consumption } \\
\text { is high. } \\
\end{array}$ & $\begin{array}{l}\checkmark \text { Continuous } \\
\text { field } \\
\text { monitoring }\end{array}$ & $\begin{array}{l}\checkmark \text { Reduced } \\
\text { water } \\
\text { consumptio } \\
\end{array}$ & $\begin{array}{l}\checkmark \text { Agricultu } \\
\text { re }\end{array}$ \\
\hline
\end{tabular}




\begin{tabular}{|c|c|c|c|c|c|c|c|c|c|}
\hline & & $\begin{array}{l}\sqrt{ } \text { Crop } \\
\text { Managem } \\
\text { ent }\end{array}$ & $\checkmark$ Light Intensity & & $\begin{array}{l}\text { the } \\
\text { irrigation } \\
\text { system. }\end{array}$ & $\begin{array}{l}\checkmark \text { High human } \\
\text { interaction. }\end{array}$ & $\begin{array}{l}\text { with the help } \\
\text { of low-cost } \\
\text { sensors. } \\
\checkmark \text { Reduces water } \\
\text { consumption. } \\
\checkmark \text { Reduced } \\
\text { power } \\
\text { consumption. } \\
\checkmark \text { Increased crop } \\
\text { productivity. } \\
\checkmark \text { Reduced } \\
\text { wastage of } \\
\text { crops. }\end{array}$ & $\begin{array}{l}\text { n up to } \\
\text { great } \\
\text { extent. }\end{array}$ & \\
\hline 30 & $\begin{array}{l}\text { Zaman et al } \\
(2016) \\
{[30]}\end{array}$ & $\begin{array}{l}\checkmark \text { Water } \\
\text { Managem } \\
\text { ent }\end{array}$ & $\begin{array}{l}\checkmark \text { Moisture level } \\
\checkmark \text { Light intensity }\end{array}$ & $\begin{array}{l}\checkmark \text { Raspberry } \\
\quad \mathrm{Pi} \\
\checkmark \text { Wi-Fi } \\
\checkmark \text { (Mobile } \\
\quad \text { Technology }\end{array}$ & $\begin{array}{l}\text { Detect } \\
\text { appropriate } \\
\text { time for } \\
\text { water } \\
\text { supply. } \\
\checkmark \text { Keep track } \\
\text { of water } \\
\text { level. }\end{array}$ & $\begin{array}{l}\checkmark \text { Cannot predict } \\
\text { the time for } \\
\text { watering. }\end{array}$ & $\begin{array}{l}\checkmark \text { Improved } \\
\text { productivity. } \\
\checkmark \text { Low cost. } \\
\checkmark \text { Utilize water } \\
\text { resources. } \\
\checkmark \text { Lessen human } \\
\text { interaction. }\end{array}$ & $\begin{array}{l}\checkmark \text { With the } \\
\text { enhanced } \\
\text { sensor } \\
\text { technology } \\
\text { will } \\
\text { become } \\
\text { more } \\
\text { efficient. }\end{array}$ & $\begin{array}{l}\checkmark \text { Agricultu } \\
\text { re }\end{array}$ \\
\hline 31 & $\begin{array}{l}\text { Biradar et al } \\
(2017) \\
{[31]}\end{array}$ & $\begin{array}{l}\checkmark \text { Water } \\
\text { Managem } \\
\text { ent } \\
\checkmark \text { Crop } \\
\text { manageme } \\
\text { nt }\end{array}$ & $\begin{array}{l}\checkmark \text { Temperature } \\
\checkmark \text { humidity } \\
\checkmark \text { Soil PH } \\
\checkmark \text { Evapotranspirati } \\
\quad \text { on }\end{array}$ & $\begin{array}{l}\checkmark \text { Wireless } \\
\text { Sensor } \\
\text { network } \\
\checkmark \text { Mobile } \\
\text { technology } \\
\checkmark \text { ZigBee } \\
\checkmark \text { RFID }\end{array}$ & $\begin{array}{l}\checkmark \text { Helps for } \\
\text { decision } \\
\text { making } \\
\text { process } \\
\checkmark \text { Can monitor } \\
\text { and control } \\
\text { the } \\
\text { temperature, } \\
\text { humidity } \\
\text { and soil PH. } \\
\checkmark \text { It can sense } \\
\text { the amount } \\
\text { of the } \\
\text { change } \\
\text { through the } \\
\text { integration } \\
\text { process of } \\
\text { components. } \\
\checkmark \text { Reduce cost }\end{array}$ & $\begin{array}{l}\checkmark \text { Unequal } \\
\text { distribution of } \\
\text { rain water } \\
\checkmark \text { Differentiation } \\
\text { of weather } \\
\text { condition. } \\
\checkmark \text { Different soil } \\
\text { types }\end{array}$ & $\begin{array}{l}\checkmark \text { Crop } \\
\text { management } \\
\text { by providing } \\
\text { required } \\
\text { amount of } \\
\text { water. } \\
\checkmark \text { Multidisciplina } \\
\text { ry monitoring } \\
\text { leads to } \\
\text { improvement } \\
\text { of agricultural } \\
\text { management. }\end{array}$ & $\begin{array}{l}\checkmark \text { Decreases } \\
\text { in the cost } \\
\text { of sensors } \\
\checkmark \text { Increasing } \\
\text { difficulty } \\
\text { of big data } \\
\text { analysis }\end{array}$ & $\begin{array}{l}\checkmark \text { Agricultu } \\
\text { re }\end{array}$ \\
\hline 32 & $\begin{array}{l}\text { Ismail et al } \\
(2017) \\
{[32]}\end{array}$ & $\begin{array}{l}\checkmark \text { Soil } \\
\text { moisture } \\
\text { level } \\
\text { monitor }\end{array}$ & $\begin{array}{l}\checkmark \text { Soil moisture } \\
\checkmark \text { Water content } \\
\checkmark \text { Temperature }\end{array}$ & $\checkmark \mathrm{Wi}-\mathrm{Fi}$ & $\begin{array}{l}\checkmark \text { Farmers can } \\
\text { face the any } \\
\text { environment } \\
\text { al } \\
\text { challenges } \\
\text { easily. } \\
\checkmark \text { Reduce the } \\
\text { harmful risk } \\
\text { percentage } \\
\checkmark \text { Save money } \\
\text { and water. } \\
\checkmark \text { Reduce pest } \\
\text { population. } \\
\end{array}$ & $\begin{array}{l}\checkmark \text { Climatic } \\
\text { change. } \\
\checkmark \text { Take long } \\
\text { time to } \\
\text { harvesting. } \\
\checkmark \text { Burnings in } \\
\text { land } \\
\text { preparation. } \\
\checkmark \text { Limitation of } \\
\text { space. }\end{array}$ & $\begin{array}{l}\checkmark \text { Monitor and } \\
\text { control the soil } \\
\text { moisture from } \\
\text { the web server. }\end{array}$ & $\begin{array}{l}\checkmark \text { Decreases } \\
\text { in the cost } \\
\text { of sensors } \\
\text { and } \\
\text { actuators. } \\
\checkmark \text { Recycling } \\
\text { the } \\
\text { resources. }\end{array}$ & $\begin{array}{l}\checkmark \text { Agricultu } \\
\text { re }\end{array}$ \\
\hline 33 & $\begin{array}{l}\text { Amandeepet } \\
\text { al (2017) } \\
{[33]}\end{array}$ & $\begin{array}{l}\checkmark \text { Machines } \\
\text { for routine } \\
\text { operations } \\
\checkmark \text { Ware } \\
\text { house } \\
\text { manageme } \\
\text { nt. }\end{array}$ & $\begin{array}{l}\checkmark \text { Soil moisture } \\
\checkmark \text { Temperature } \\
\checkmark \text { Humidity } \\
\checkmark \text { Water level }\end{array}$ & $\begin{array}{l}\checkmark \text { ZigBee } \\
\checkmark \text { Mobile } \\
\text { technology } \\
\checkmark \text { Wi-fi }\end{array}$ & $\begin{array}{l}\checkmark \text { Increasing } \\
\text { the crop } \\
\text { productivity } \\
\text {. } \\
\checkmark \text { Prevent } \\
\text { thefts. } \\
\checkmark \text { Prevent } \\
\text { attacking } \\
\text { from birds, } \\
\text { animals and } \\
\text { other facts. }\end{array}$ & $\begin{array}{l}\checkmark \text { Manual } \\
\text { distribution of } \\
\text { seeds. } \\
\checkmark \text { Pattern of two } \\
\text { crops year. } \\
\checkmark \text { Unscientific } \\
\text { system of } \\
\text { cultivation. } \\
\checkmark \text { Unequal } \\
\text { watering } \\
\text { system. }\end{array}$ & $\begin{array}{l}\checkmark \text { Using remote } \\
\text { control vehicle } \\
\text { keeps } \\
\text { monitoring the } \\
\text { humidity, soil } \\
\text { condition and } \\
\text { water level in } \\
\text { the field. }\end{array}$ & $\begin{array}{l}\checkmark \text { Improve } \\
\text { the green } \\
\text { energy } \\
\text { concept for } \\
\text { better } \\
\text { productivit } \\
\text { y }\end{array}$ & $\checkmark$ Farming \\
\hline 34 & $\begin{array}{l}\text { Dolci (2017) } \\
\text { [34] }\end{array}$ & $\begin{array}{l}\checkmark \text { Precision } \\
\text { farming } \\
\checkmark \text { Prescriptiv } \\
\text { e farming }\end{array}$ & $\begin{array}{l}\checkmark \text { Temperature } \\
\checkmark \text { humidity } \\
\checkmark \text { Soil PH } \\
\checkmark \text { CO2 }\end{array}$ & $\begin{array}{l}\checkmark \text { Mobile } \\
\quad \text { technology }\end{array}$ & $\begin{array}{l}\checkmark \text { Cost } \\
\text { reduction } \\
\checkmark \text { Reduce the } \\
\text { frequency }\end{array}$ & $\begin{array}{l}\checkmark \text { Unequal } \\
\text { distribution of } \\
\text { air flow. }\end{array}$ & $\begin{array}{l}\checkmark \text { Improving } \\
\text { malt quality } \\
\text { and efficiency } \\
\text { in production } \\
\text { with using } \\
\text { Artificial } \\
\text { Intelligence }\end{array}$ & $\begin{array}{l}\checkmark \text { low cost } \\
\text { sensors } \\
\checkmark \text { open } \\
\text { source } \\
\checkmark \text { application } \\
\text { s } \\
\checkmark \text { ability to } \\
\text { increase the } \\
\text { level of } \\
\text { farming } \\
\end{array}$ & $\checkmark$ Farming \\
\hline
\end{tabular}




\begin{tabular}{|c|c|c|c|c|c|c|c|c|c|}
\hline & & & & & & & & $\begin{array}{l}\text { sophisticati } \\
\text { on }\end{array}$ & \\
\hline 35 & $\begin{array}{l}\text { Gokul et al } \\
(2017) \\
{[35]}\end{array}$ & $\begin{array}{l}\checkmark \text { Livestock } \\
\text { manageme } \\
\text { nt } \\
\checkmark \text { Smart } \\
\text { lightening } \\
\checkmark \text { Smart } \\
\text { ventilation } \\
\checkmark \text { Water } \\
\text { manageme } \\
\text { nt }\end{array}$ & $\begin{array}{l}\checkmark \text { Temperature } \\
\checkmark \text { humidity } \\
\checkmark \text { Milk production }\end{array}$ & $\begin{array}{l}\checkmark \text { Mobile } \\
\text { technology } \\
\checkmark \text { Wi-Fi }\end{array}$ & $\begin{array}{l}\checkmark \text { Identifying } \\
\text { the } \\
\text { emergency } \\
\text { conditions. } \\
\checkmark \text { Improves } \\
\text { location } \\
\text { tracking. } \\
\checkmark \text { Improves } \\
\text { cattle health } \\
\checkmark \text { Improves } \\
\text { availability }\end{array}$ & $\begin{array}{l}\checkmark \text { Unable to } \\
\text { detect illnesses } \\
\text { early. } \\
\checkmark \text { Different } \\
\text { environmental } \\
\text { conditions } \\
\checkmark \text { Irregular } \\
\text { feeding }\end{array}$ & $\begin{array}{l}\checkmark \text { Make } \\
\checkmark \text { Infrastructure } \\
\text { of cattle } \\
\text { farming } \\
\text { smarter. } \\
\checkmark \text { implement a } \\
\text { noninvasive } \\
\checkmark \text { wearable to } \\
\text { track } \\
\text { physiological } \\
\text { and biological } \\
\text { activities of } \\
\text { cattle. }\end{array}$ & $\begin{array}{l}\checkmark \text { Improve } \\
\text { the smart } \\
\text { lightning } \\
\text { and } \\
\text { ventilation } \\
\text { system }\end{array}$ & $\begin{array}{l}\checkmark \text { Farming, } \\
\text { Agricultu } \\
\text { re }\end{array}$ \\
\hline 36 & $\begin{array}{l}\text { Rajkumar et } \\
\text { al (2017) } \\
{[36]}\end{array}$ & $\begin{array}{l}\checkmark \text { Irrigation } \\
\text { manageme } \\
\text { nt }\end{array}$ & $\begin{array}{l}\checkmark \text { Temperature } \\
\checkmark \text { humidity } \\
\checkmark \text { Soil Moisture }\end{array}$ & $\begin{array}{l}\checkmark \text { Mobile } \\
\text { technology } \\
\checkmark \text { Wi-Fi }\end{array}$ & $\begin{array}{l}\checkmark \text { Provides } \\
\text { real time } \\
\text { information } \\
\checkmark \text { Cost } \\
\text { reduction } \\
\checkmark \text { Resource } \\
\text { optimization } \\
\checkmark \text { Reduce } \\
\text { water } \\
\text { logging and } \\
\text { shortage }\end{array}$ & $\begin{array}{l}\checkmark \text { Water } \\
\text { shortage } \\
\checkmark \text { Different } \\
\text { environmental } \\
\text { conditions }\end{array}$ & $\begin{array}{l}\checkmark \\
\checkmark \text { Developing } \\
\text { smart irrigation } \\
\text { system to } \\
\text { monitor at } \\
\text { anywhere. }\end{array}$ & $\begin{array}{l}\checkmark \text { Installing a } \\
\text { water meter } \\
\text { to estimate } \\
\text { the amount } \\
\text { of water. } \\
\checkmark \text { Using } \\
\text { Wireless } \\
\text { sensors. }\end{array}$ & $\begin{array}{l}\checkmark \text { Agricultu } \\
\quad \text { re }\end{array}$ \\
\hline $\begin{array}{l}37 \\
.\end{array}$ & $\begin{array}{l}\text { Sri et al } \\
(2017) \\
{[37]}\end{array}$ & $\begin{array}{l}\checkmark \text { Crop } \\
\text { manageme } \\
\text { nt } \\
\checkmark \text { Irrigation } \\
\quad \text { manageme } \\
\text { nt }\end{array}$ & $\begin{array}{l}\checkmark \text { Soil } \\
\checkmark \text { Temperature } \\
\checkmark \text { Humidity } \\
\checkmark \text { Rain fall } \\
\checkmark \text { Fertilizer } \\
\quad \text { efficiency }\end{array}$ & $\begin{array}{l}\checkmark \text { Wi-Fi } \\
\checkmark \text { GPRS } \\
\checkmark \text { Zig Bee } \\
\checkmark \text { Raspberry } \\
\quad \text { pi } \\
\checkmark \text { Mobile } \\
\text { technology }\end{array}$ & $\begin{array}{l}\checkmark \\
\checkmark \text { Improve the } \\
\text { yield } \\
\checkmark \text { Low cost }\end{array}$ & $\begin{array}{l}\checkmark \text { Unpredictable } \\
\text { weather } \\
\checkmark \text { Water scarcity } \\
\checkmark \text { Improper } \\
\quad \text { water usage }\end{array}$ & $\begin{array}{l}\checkmark \\
\checkmark \text { Providing } \\
\text { reliable and } \\
\text { efficient } \\
\text { agricultural } \\
\text { system to } \\
\text { monitor the } \\
\text { field }\end{array}$ & $\begin{array}{l}\checkmark \text { Improve } \\
\checkmark \text { by adding } \\
\text { several } \\
\text { modern } \\
\text { techniques } \\
\text { like } \\
\text { irrigation } \\
\checkmark \text { Method, } \\
\text { solar power } \\
\text { source } \\
\text { usage. }\end{array}$ & $\begin{array}{l}\checkmark \text { Agricultu } \\
\text { re }\end{array}$ \\
\hline $\begin{array}{l}38 \\
\cdot\end{array}$ & $\begin{array}{l}\text { Rajarsri et al } \\
(2017) \\
{[38]}\end{array}$ & $\begin{array}{l}\checkmark \text { Water } \\
\text { manageme } \\
\text { nt }\end{array}$ & $\checkmark$ Water level & $\begin{array}{l}\checkmark \text { Mobile } \\
\text { technology }\end{array}$ & $\begin{array}{l}\checkmark \text { Improve the } \\
\text { efficiency } \\
\checkmark \text { Optimize } \\
\text { resource } \\
\checkmark \text { Maximize } \\
\text { the profit }\end{array}$ & $\begin{array}{l}\checkmark \text { Unequal water } \\
\text { distribution }\end{array}$ & $\begin{array}{l}\checkmark \text { Build a well- } \\
\text { connected } \\
\text { farming } \\
\text { network and } \\
\text { create a } \\
\text { knowledge } \\
\text { sharing } \\
\text { platform. }\end{array}$ & $\begin{array}{l}\checkmark \text { Agro loan } \\
\checkmark \text { Inexpensiv } \\
\text { e } \\
\text { Agricultura } \\
1 \\
\text { consultatio } \\
n \\
\checkmark \text { better ROI } \\
\checkmark \text { Agro } \\
\text { networking } \\
\checkmark \text { Low cost } \\
\text { products }\end{array}$ & $\begin{array}{l}\checkmark \text { Agricultu } \\
\quad \text { re }\end{array}$ \\
\hline $\begin{array}{l}39 \\
.\end{array}$ & $\begin{array}{l}\text { Ruengittinun } \\
\text { et al (2017) } \\
{[39]}\end{array}$ & $\begin{array}{l}\checkmark \text { Smart } \\
\text { farming }\end{array}$ & $\begin{array}{l}\checkmark \text { Temperature } \\
\checkmark \text { Humidity } \\
\checkmark \text { PH } \\
\checkmark \text { Electrical } \\
\quad \text { conductivity }\end{array}$ & $\checkmark$ Wi-Fi & $\begin{array}{l}\checkmark \text { Can farm in } \\
\text { less space } \\
\checkmark \text { Provides } \\
\text { many } \\
\text { products }\end{array}$ & $\begin{array}{l}\checkmark \text { Differential of } \\
\text { temperature } \\
\checkmark \text { Lack of time } \\
\text { to manage and } \\
\text { plant. }\end{array}$ & $\begin{array}{l}\checkmark \text { Build a smart } \\
\text { hydroponic eco } \\
\text { system }\end{array}$ & $\begin{array}{l}\checkmark \text { Symmetric } \\
\text { al } \\
\checkmark \text { plantation } \\
\text { to check } \\
\text { the } \\
\text { accuracy of } \\
\text { the HFE } \\
\text { across } \\
\text { multiple } \\
\checkmark \text { farms in the } \\
\text { same area. }\end{array}$ & $\checkmark$ Farming \\
\hline
\end{tabular}




\begin{tabular}{|c|c|c|c|c|c|c|c|c|c|}
\hline $\begin{array}{l}40 \\
.\end{array}$ & $\begin{array}{c}\checkmark \text { Yoon et al } \\
(2018) \\
\checkmark[40]\end{array}$ & $\begin{array}{l}\checkmark \text { Smart } \\
\text { farming } \\
\checkmark \text { Irrigation } \\
\text { manageme } \\
\text { nt }\end{array}$ & $\begin{array}{l}\checkmark \text { Temperature } \\
\checkmark \text { Humidity } \\
\checkmark \text { CO2 } \\
\checkmark\end{array}$ & $\begin{array}{l}\checkmark \text { Bluetooth } \\
\checkmark \text { Wi-Fi } \\
\checkmark \text { LPWAN } \\
\checkmark\end{array}$ & $\begin{array}{l}\checkmark \checkmark \text { can } \\
\text { overcome } \\
\text { distance } \\
\checkmark \text { and place } \\
\text { constraints. } \\
\checkmark \text { save } \\
\text { maintenance } \\
\text { cost of } \\
\text { existing } \\
\text { devices } \\
\checkmark \text { provide } \\
\checkmark \text { compatibilit } \\
\text { y with new } \\
\text { devices }\end{array}$ & $\begin{array}{l}\checkmark \text { Power } \\
\text { problem } \\
\checkmark \text { Space } \\
\text { limitation } \\
\checkmark \text { difficulties in } \\
\text { installing } \\
\text { additional } \\
\text { devices }\end{array}$ & $\begin{array}{l}\checkmark \text { Build a system } \\
\text { with using } \\
\text { Bluetooth and } \\
\text { LPWAN to } \\
\text { solve the } \\
\text { power problem } \\
\text { and space } \\
\text { limitation. }\end{array}$ & $\begin{array}{l}\checkmark \text { System for } \\
\text { studying } \\
\text { the } \\
\text { developme } \\
\text { nt of } \\
\text { environmen } \\
\text { tal } \\
\checkmark \text { Algorithms } \\
.\end{array}$ & $\begin{array}{l}\checkmark \text { Farming, } \\
\text { Agricultu } \\
\text { re }\end{array}$ \\
\hline 41 & $\begin{array}{l}\text { Ezhilazhahi } \\
\text { et al (2017) } \\
{[41]}\end{array}$ & $\begin{array}{l}\checkmark \text { Smart } \\
\quad \text { Farming }\end{array}$ & $\begin{array}{l}\checkmark \text { Plant health } \\
\checkmark \text { Soil Moisture } \\
\checkmark \text { Temperature } \\
\checkmark \text { Humidity }\end{array}$ & $\begin{array}{l}\checkmark \text { WSN } \\
\checkmark \text { Zig Bee } \\
\checkmark \text { Wi-Fi } \\
\checkmark \text { Raspberry } \\
\text { pi } \\
\checkmark \text { GPRS }\end{array}$ & $\begin{array}{l}\checkmark \text { Enrich the } \\
\text { productivity } \\
\text { of food } \\
\text { grains. } \\
\checkmark \text { Prevent the } \\
\text { plant from } \\
\text { blight and } \\
\text { harmful } \\
\checkmark \text { insects. }\end{array}$ & $\begin{array}{l}\checkmark \text { water scarcity } \\
\checkmark \text { unpredictable } \\
\text { weather } \\
\text { conditions }\end{array}$ & $\begin{array}{l}\checkmark \text { Developing a } \\
\text { system to } \\
\text { monitor } \\
\text { continuously } \\
\text { soil moisture } \\
\text { of the plants. }\end{array}$ & $\begin{array}{l}\checkmark \text { Increasing } \\
\text { the number } \\
\text { of sensors. }\end{array}$ & $\checkmark$ Farming \\
\hline 42 & $\begin{array}{l}\text { Tanmayee } \\
(2017) \\
{[42]}\end{array}$ & $\begin{array}{l}\checkmark \text { Crop } \\
\text { manageme } \\
\text { nt }\end{array}$ & $\begin{array}{l}\checkmark \text { Temperature } \\
\checkmark \text { Soil Moisture }\end{array}$ & $\begin{array}{l}\checkmark \text { Raspberry } \\
\text { pi } \\
\checkmark \text { Mobile } \\
\text { technology }\end{array}$ & $\begin{array}{l}\checkmark \text { Reduces the } \\
\text { wastage of } \\
\text { pesticides } \\
\checkmark \text { Reduces the } \\
\text { human } \\
\text { effort } \\
\checkmark \text { Increase } \\
\text { agricultural } \\
\text { productivity }\end{array}$ & $\begin{array}{l}\checkmark \text { Bacterial } \\
\text { diseases } \\
\checkmark \text { unpredictable } \\
\text { weather } \\
\text { conditions }\end{array}$ & $\begin{array}{l}\checkmark \text { Implementing } \\
\text { a rice crop } \\
\text { monitoring } \\
\text { System }\end{array}$ & $\begin{array}{l}\checkmark \text { Using } \\
\text { multicolor } \\
\text { detection } \\
\text { for detect } \\
\text { the disease } \\
\text { in any } \\
\text { stage. }\end{array}$ & $\begin{array}{l}\checkmark \text { Agricultu } \\
\text { re }\end{array}$ \\
\hline 43 & $\begin{array}{l}\text { Takecar et al } \\
(2017) \\
{[43]}\end{array}$ & $\begin{array}{l}\checkmark \text { Machines } \\
\text { for routine } \\
\text { operations }\end{array}$ & $\begin{array}{l}\checkmark \text { Soil Moisture } \\
\checkmark \text { Temperature } \\
\checkmark \text { Humidity }\end{array}$ & $\checkmark$ Wi-fi. & $\begin{array}{l}\checkmark \text { Increase the } \\
\text { income } \\
\checkmark \text { Cost } \\
\text { reduction }\end{array}$ & $\begin{array}{l}\checkmark \text { Lack of } \\
\text { Resource } \\
\text { Management }\end{array}$ & $\begin{array}{l}\checkmark \text { Implementing } \\
\text { a system to } \\
\text { look after the } \\
\text { plantation } \\
\text { without } \\
\text { disturbing busy } \\
\text { schedule. }\end{array}$ & $\begin{array}{l}\checkmark \text { Improve } \\
\text { the } \\
\text { component } \\
\text { s in the } \\
\text { PATRIOT } \\
\text { system }\end{array}$ & $\checkmark$ Farming \\
\hline 44 & $\begin{array}{l}\text { Krishna et al } \\
(2017) \\
{[44]}\end{array}$ & $\begin{array}{l}\checkmark \text { Smart } \\
\text { Farming } \\
\checkmark \text { livestock } \\
\text { manageme } \\
\text { nt }\end{array}$ & $\begin{array}{l}\checkmark \text { Soil Moisture } \\
\checkmark \text { Light intensity } \\
\checkmark \text { Humidity } \\
\checkmark \text { Temperature } \\
\checkmark \text { Soil pH }\end{array}$ & $\begin{array}{l}\checkmark \text { Raspberry } \\
\text { pi } \\
\checkmark \text { Zig Bee } \\
\checkmark \text { Wi-Fi }\end{array}$ & $\begin{array}{l}\checkmark \text { Reducing } \\
\text { labor costs } \\
\checkmark \text { Helps to } \\
\checkmark \text { track the } \\
\text { changes } \\
\text { accurately } \\
\text { occurring } \\
\text { instantly in } \\
\text { real time at } \\
\text { the field. }\end{array}$ & $\begin{array}{l}\checkmark \text { lack of } \\
\text { moisture in the } \\
\text { fields } \\
\checkmark \text { salinity } \\
\checkmark \text { lack of } \\
\text { application of } \\
\text { fertilizers } \\
\checkmark \text { Different } \\
\text { sowing time. }\end{array}$ & $\begin{array}{l}\checkmark \text { Using wireless } \\
\text { mobile robot } \\
\text { performing } \\
\text { various } \\
\text { operations of } \\
\text { the field. }\end{array}$ & $\begin{array}{l}\checkmark \text { Develop } \\
\text { the } \\
\text { capabilities } \\
\text { of the } \\
\text { robot. }\end{array}$ & $\checkmark$ Farming \\
\hline 45 & $\begin{array}{l}\text { Li et al } \\
(2017) \\
{[45]}\end{array}$ & $\begin{array}{l}\checkmark \text { Irrigation } \\
\text { manageme } \\
\text { nt } \\
\checkmark \text { Greenhous } \\
\text { e } \\
\text { manageme } \\
\text { nt }\end{array}$ & $\begin{array}{l}\checkmark \text { Humidity } \\
\checkmark \text { Temperature }\end{array}$ & $\begin{array}{l}\checkmark \text { Zig Bee } \\
\checkmark \text { Wi-Fi } \\
\checkmark \text { Bluetooth } \\
\checkmark \text { LAN }\end{array}$ & $\begin{array}{l}\checkmark \text { Reduce the } \\
\text { labour cost. } \\
\checkmark \text { Improve the } \\
\text { efficiency of } \\
\text { agricultural } \\
\text { production. }\end{array}$ & $\begin{array}{l}\checkmark \text { low } \\
\text { production } \\
\text { efficiency } \\
\checkmark \text { Waste of } \\
\checkmark \text { resources } \\
\checkmark \text { Environmental } \\
\text { pollution. }\end{array}$ & $\begin{array}{l}\checkmark \text { Green house } \\
\text { management to } \\
\text { improve the } \\
\text { agricultural } \\
\text { production. }\end{array}$ & $\begin{array}{l}\checkmark \text { Implement } \\
\text { a } \\
\text { comprehen } \\
\text { sive } \\
\text { promotion } \\
\text { system. }\end{array}$ & $\begin{array}{l}\checkmark \text { Agricultu } \\
\text { re }\end{array}$ \\
\hline 46 & $\begin{array}{l}\text { Suciu et al } \\
(2016) \\
{[46]}\end{array}$ & $\begin{array}{l}\checkmark \text { Smart } \\
\quad \text { Farming }\end{array}$ & $\checkmark$ Temperature & $\begin{array}{l}\checkmark \text { Mobile } \\
\text { technology } \\
\checkmark \text { GPRS }\end{array}$ & $\begin{array}{l}\checkmark \text { Improve the } \\
\text { quality and } \\
\text { safety of the } \\
\text { products } \\
\checkmark \text { Detecting } \\
\text { plant } \\
\text { diseases, } \\
\text { flood. Etc. }\end{array}$ & $\begin{array}{l}\checkmark \text { Climatic } \\
\text { change } \\
\checkmark \text { High } \\
\text { temperature } \\
\checkmark \text { Low profit } \\
\text { margin }\end{array}$ & $\begin{array}{l}\checkmark \text { Assist for crop } \\
\text { management } \\
\text { by using smart } \\
\text { agriculture }\end{array}$ & $\begin{array}{l}\text { Allowing } \\
\text { system to } \\
\text { measure } \\
\text { basic } \\
\text { parameters } \\
\text { for } \\
\text { irrigation } \\
\text { manageme } \\
\text { nt. } \\
\end{array}$ & $\checkmark$ Farming \\
\hline 47 & $\begin{array}{l}\text { Putjaika et al } \\
(2017) \\
{[47]}\end{array}$ & $\begin{array}{l}\checkmark \text { Intelligent } \\
\text { farming }\end{array}$ & $\begin{array}{l}\checkmark \text { Humidity } \\
\checkmark \text { Temperature } \\
\checkmark \text { Soil moisture } \\
\checkmark \text { Light intensity }\end{array}$ & $\checkmark \mathrm{Wi}-\mathrm{Fi}$ & $\begin{array}{l}\checkmark \text { Improve the } \\
\text { production } \\
\text { process } \\
\checkmark \text { Managing } \\
\text { resources }\end{array}$ & $\begin{array}{l}\checkmark \text { Unpredictable } \\
\text { weather }\end{array}$ & $\begin{array}{l}\checkmark \text { Implement a } \\
\text { system to } \\
\text { monitor the } \\
\text { harmful } \\
\text { diseases. }\end{array}$ & $\begin{array}{l}\checkmark \text { Developing } \\
\text { the sensor } \\
\text { and control } \\
\text { system by } \\
\text { adding } \\
\text { more } \\
\text { component } \\
\text { s. }\end{array}$ & $\checkmark$ Farming \\
\hline
\end{tabular}




\begin{tabular}{|c|c|c|c|c|c|c|c|c|c|}
\hline 48 & $\begin{array}{l}\text { Okayasu et al } \\
(2017) \\
{[48]}\end{array}$ & $\begin{array}{l}\checkmark \text { Growth } \\
\text { measurem } \\
\text { ent }\end{array}$ & $\begin{array}{l}\checkmark \text { Humidity } \\
\checkmark \text { Temperature } \\
\checkmark \text { Solar radiation } \\
\checkmark \text { CO2 }\end{array}$ & $\checkmark \mathrm{Wi}-\mathrm{Fi}$ & $\begin{array}{l}\checkmark \text { Reduce } \\
\text { production } \\
\text { cost } \\
\checkmark \text { Improve the } \\
\text { quality of } \\
\text { the products }\end{array}$ & $\begin{array}{l}\checkmark \text { High } \\
\text { production } \\
\text { cost } \\
\checkmark \text { Less quality in } \\
\text { products }\end{array}$ & $\begin{array}{l}\checkmark \text { Monitoring the } \\
\text { plant growth } \\
\text { measurement } \\
\text { using smart } \\
\text { agriculture. }\end{array}$ & $\begin{array}{l}\checkmark \text { Improve } \\
\text { the } \\
\text { accuracy of } \\
\text { measureme } \\
\text { nts. }\end{array}$ & $\checkmark$ Farming \\
\hline 49 & $\begin{array}{l}\text { Sreekantha et } \\
\text { al (2017) } \\
{[49]}\end{array}$ & $\begin{array}{l}\checkmark \text { Irrigation } \\
\text { manageme } \\
\text { nt. } \\
\checkmark \text { Greenhous } \\
\text { e } \\
\text { manageme } \\
\text { nt }\end{array}$ & $\begin{array}{l}\checkmark \text { Temperature } \\
\checkmark \text { Soil moisture } \\
\checkmark \text { Weather } \\
\checkmark \text { Fertility of soil }\end{array}$ & $\begin{array}{l}\checkmark \text { Zig Bee } \\
\checkmark \text { Mobile } \\
\text { technology } \\
\checkmark \text { Wi-Fi }\end{array}$ & $\begin{array}{l}\checkmark \text { Detection of } \\
\text { seed, water } \\
\text { level, pest, } \\
\text { animal } \\
\text { intrusion to } \\
\text { the field. } \\
\checkmark \text { Reduce cost } \\
\text { and time } \\
\checkmark \text { Enhance } \\
\text { productivity }\end{array}$ & $\begin{array}{l}\checkmark \text { Environmental } \\
\text { changes } \\
\checkmark \text { High water } \\
\text { consumption }\end{array}$ & $\begin{array}{l}\checkmark \text { Enhance the } \\
\text { productivity by } \\
\text { using crop } \\
\text { monitoring } \\
\text { system. }\end{array}$ & $\begin{array}{l}\checkmark \text { generalize } \\
\text { event- } \\
\text { condition- } \\
\text { action } \\
\checkmark \text { framework } \\
\quad \text { for } \\
\text { programmi } \\
\text { ng reactive } \\
\text { sensor } \\
\text { networks }\end{array}$ & $\begin{array}{l}\checkmark \text { Agricultu } \\
\text { re }\end{array}$ \\
\hline 50 & $\begin{array}{l}\text { Rajendrakum } \\
\text { ar et al } \\
(2017) \\
{[50]}\end{array}$ & $\begin{array}{l}\checkmark \text { Water } \\
\text { manageme } \\
\text { nt } \\
\checkmark \text { Crop } \\
\text { manageme } \\
\text { nt }\end{array}$ & $\begin{array}{l}\checkmark \text { Soil moisture } \\
\checkmark \text { Temperature } \\
\checkmark \text { Humidity } \\
\checkmark \text { Soil pH }\end{array}$ & $\begin{array}{l}\checkmark \text { Mobile } \\
\text { technology } \\
\checkmark \text { Wi-Fi }\end{array}$ & $\begin{array}{l}\checkmark \text { Increase } \\
\text { harvest } \\
\text { efficiency } \\
\checkmark \text { Decrease } \\
\text { water } \\
\text { wastage }\end{array}$ & $\begin{array}{l}\checkmark \text { Uncertain } \\
\text { monsoon } \\
\checkmark \text { Water scarcity } \\
\checkmark \text { Climatic } \\
\quad \text { variation }\end{array}$ & $\begin{array}{l}\checkmark \text { Providing } \\
\text { information to } \\
\text { understand } \\
\text { how to monitor } \\
\text { and control the } \\
\text { data remotely } \\
\text { and apply to } \\
\text { the fields. }\end{array}$ & $\begin{array}{r}\checkmark \text { Develop } \\
\text { multiple } \\
\text { systems. }\end{array}$ & $\begin{array}{l}\checkmark \text { Agricultu } \\
\text { re }\end{array}$ \\
\hline 51 & $\begin{array}{l}\text { Ferreira et al } \\
(2017) \\
{[51]}\end{array}$ & $\begin{array}{l}\checkmark \text { Smart } \\
\text { Farming } \\
\checkmark \text { Machines } \\
\text { for routine } \\
\text { operations }\end{array}$ & $\begin{array}{l}\checkmark \text { Temperature } \\
\checkmark \text { Soil pH } \\
\checkmark \text { Oxygen flow }\end{array}$ & $\begin{array}{l}\checkmark \text { Mobile } \\
\text { technology }\end{array}$ & $\begin{array}{l}\checkmark \text { Improve the } \\
\text { production. }\end{array}$ & $\begin{array}{l}\checkmark \text { Climate } \\
\text { changes. } \\
\checkmark \text { Insufficient } \\
\text { available } \\
\text { lands. } \\
\checkmark \text { Air toxins. }\end{array}$ & $\begin{array}{l}\checkmark \text { Researching } \\
\text { modules } \\
\checkmark \text { related to IoT, } \\
\text { event } \\
\text { processing, } \\
\text { situational } \\
\text { awareness and } \\
\text { data } \\
\checkmark \text { harmonization }\end{array}$ & $\begin{array}{l}\checkmark \text { Developing } \\
\text { all the apps } \\
\text { and } \\
\text { experiment } \\
\text { with real } \\
\text { cases. }\end{array}$ & $\checkmark$ Farming \\
\hline 52 & $\begin{array}{l}\text { Vernandhes } \\
\text { et al (2017) } \\
{[52]}\end{array}$ & $\begin{array}{l}\checkmark \text { Livestock } \\
\text { manageme } \\
\text { nt }\end{array}$ & $\begin{array}{l}\checkmark \text { Temperature } \\
\checkmark \text { Humidity } \\
\checkmark \text { Light }\end{array}$ & $\begin{array}{l}\checkmark \text { Mobile } \\
\text { technology } \\
\checkmark \text { Wi-Fi }\end{array}$ & $\begin{array}{l}\checkmark \text { Improve the } \\
\text { cultivation }\end{array}$ & $\begin{array}{l}\checkmark \text { Limited lands. } \\
\checkmark \text { Water scarcity }\end{array}$ & $\begin{array}{l}\text { Smart } \\
\text { aquaponic } \\
\text { system to } \\
\text { monitor and } \\
\text { control } \\
\text { cultivation } \\
\end{array}$ & $\begin{array}{l}\checkmark \text { Increase } \\
\text { the manual } \\
\text { response } \\
\text { speed. }\end{array}$ & $\checkmark$ Farming \\
\hline 53 & $\begin{array}{l}\text { Vaughan et al } \\
(2017) \\
{[53]}\end{array}$ & $\begin{array}{l}\checkmark \text { Livestock } \\
\text { manageme } \\
\text { nt. } \\
\checkmark \text { Farm } \\
\text { manageme } \\
\text { nt. }\end{array}$ & $\checkmark$ Animal Weight & $\begin{array}{l}\checkmark \text { Mobile } \\
\text { technology }\end{array}$ & $\begin{array}{l}\checkmark \text { Can monitor } \\
\text { the } \\
\text { performance } \\
\text { of their } \\
\text { animals. } \\
\checkmark \text { Improve the } \\
\text { livestock } \\
\text { production }\end{array}$ & $\begin{array}{l}\checkmark \text { Weather } \\
\text { condition } \\
\checkmark \text { Maintaining } \\
\text { balance } \\
\checkmark \text { Large number } \\
\quad \text { of } \\
\text { measurements. }\end{array}$ & $\begin{array}{l}\checkmark \text { Gaining data } \\
\text { under the } \\
\text { hostile } \\
\text { conditions of a } \\
\text { livestock farm. }\end{array}$ & $\begin{array}{l}\checkmark \text { Upstream } \\
\text { and } \\
\text { downstrea } \\
\text { m the } \\
\text { supply } \\
\text { chain. }\end{array}$ & $\checkmark$ Farming \\
\hline 54 & $\begin{array}{l}\text { Padalalu et al } \\
(2017) \\
{[54]}\end{array}$ & $\begin{array}{l}\checkmark \text { Water } \\
\text { manageme } \\
\text { nt }\end{array}$ & $\begin{array}{l}\checkmark \text { Temperature } \\
\checkmark \text { Humidity } \\
\checkmark \text { Light } \\
\checkmark \text { CO2 } \\
\checkmark \text { Soil pH }\end{array}$ & $\begin{array}{l}\checkmark \text { Mobile } \\
\text { technology }\end{array}$ & $\begin{array}{l}\checkmark \text { Conserve } \\
\text { water } \\
\checkmark \text { Avoidance } \\
\text { of constant } \\
\text { vigilance. } \\
\checkmark \text { Remote } \\
\text { automation }\end{array}$ & $\begin{array}{l}\checkmark \text { Water scarcity } \\
\checkmark \text { High power } \\
\text { consumption }\end{array}$ & $\begin{array}{l}\checkmark \text { Implementing } \\
\text { system to to } \\
\text { make the } \\
\checkmark \text { irrigation } \\
\text { system smart, } \\
\text { autonomous } \\
\text { and efficient }\end{array}$ & $\begin{array}{l}\checkmark \text { Estimate } \\
\text { the } \\
\text { irrigation } \\
\text { cost. } \\
\checkmark \text { Introducing } \\
\text { wireless } \\
\text { sensor. } \\
\checkmark \text { Automatic } \\
\text { watering } \\
\end{array}$ & $\begin{array}{l}\checkmark \text { Agricultu } \\
\quad \text { re }\end{array}$ \\
\hline 55 & $\begin{array}{l}\text { Bellini et al } \\
(2017) \\
{[55]}\end{array}$ & $\begin{array}{l}\checkmark \text { Cattle } \\
\text { detection } \\
\text { manageme } \\
\text { nt }\end{array}$ & $\begin{array}{l}\checkmark \text { Temperature } \\
\checkmark \text { Milk } \\
\quad \text { consumption }\end{array}$ & $\checkmark$ LoRa & $\begin{array}{l}\checkmark \text { Increase } \\
\text { milk } \\
\text { production }\end{array}$ & $\begin{array}{l}\checkmark \text { Heat detection } \\
\checkmark \text { Intensification } \\
\text { management } \\
\text { techniques }\end{array}$ & $\begin{array}{l}\checkmark \text { By collecting } \\
\text { activity data } \\
\text { for heat } \\
\text { detection for } \\
\text { the cattle. }\end{array}$ & $\begin{array}{l}\checkmark \text { Developing } \\
\text { power } \\
\text { reduction } \\
\text { systems. }\end{array}$ & $\checkmark$ Farming \\
\hline 56 & $\begin{array}{l}\text { Cambra et al } \\
(2017) \\
{[56]}\end{array}$ & $\begin{array}{l}\checkmark \text { Energy } \\
\text { manageme } \\
\text { nt } \\
\checkmark \text { Water } \\
\text { manageme } \\
\text { nt }\end{array}$ & $\begin{array}{l}\checkmark \text { Temperature } \\
\checkmark \text { Humidity }\end{array}$ & $\begin{array}{l}\checkmark \text { WSN } \\
\checkmark \text { Mobile } \\
\text { technology } \\
\checkmark \text { LoRa } \\
\checkmark \text { Zig Bee }\end{array}$ & $\begin{array}{l}\checkmark \text { Energy } \\
\text { efficiency } \\
\checkmark \text { Reduction } \\
\text { in fertilizers } \\
\text { in products } \\
\checkmark \text { Saving } \\
\text { water }\end{array}$ & $\begin{array}{l}\checkmark \text { Scalability } \\
\checkmark \text { Manageability }\end{array}$ & $\begin{array}{l}\checkmark \text { Implement a } \\
\text { smart } \\
\text { communication } \\
\text { system to } \\
\text { monitor the } \\
\text { agriculture }\end{array}$ & $\begin{array}{l}\checkmark \text { Developing } \\
\text { irrigation } \\
\text { services } \\
\text { system in } \\
\text { the domain } \\
\text { of } \\
\text { agricultural } \\
\text { decision } \\
\text { systems } \\
\end{array}$ & $\begin{array}{l}\checkmark \text { Agricultu } \\
\quad \text { re }\end{array}$ \\
\hline 57 & $\begin{array}{l}\text { Moon et al } \\
(2017) \\
{[57]}\end{array}$ & $\begin{array}{l}\checkmark \text { Smart } \\
\text { farming }\end{array}$ & $\begin{array}{l}\checkmark \text { Temperature } \\
\checkmark \text { Humidity } \\
\checkmark \text { Rain fall } \\
\checkmark \text { Wind speed }\end{array}$ & $\checkmark \mathrm{Wi}-\mathrm{Fi}$ & $\begin{array}{l}\checkmark \text { Improve } \\
\text { crop yield. } \\
\checkmark \quad \text { Reduce } \\
\text { unnecessary }\end{array}$ & $\begin{array}{l}\checkmark \text { Managing big } \\
\text { data }\end{array}$ & $\begin{array}{l}\checkmark \text { Applying lossy } \\
\text { compression } \\
\text { on IoT } \\
\checkmark \text { big data. }\end{array}$ & $\begin{array}{l}\checkmark \text { Use lossy } \\
\text { compressio } \\
\mathrm{n} \\
\text { techniques }\end{array}$ & $\checkmark$ Farming \\
\hline
\end{tabular}




\begin{tabular}{|c|c|c|c|c|c|c|c|c|c|}
\hline & & & & & costs. & & & $\begin{array}{l}\checkmark \text { to reduce } \\
\text { the high } \\
\text { cost of data } \\
\text { storage and } \\
\text { transit }\end{array}$ & \\
\hline 58 & $\begin{array}{l}\text { Raghudathes } \\
\text { h et al (2017) } \\
{[58]}\end{array}$ & $\begin{array}{l}\checkmark \text { Poultry } \\
\text { manageme } \\
\text { nt }\end{array}$ & $\begin{array}{l}\checkmark \text { Temperature } \\
\checkmark \text { Humidity } \\
\checkmark \text { Light intensity } \\
\checkmark \text { Air quality }\end{array}$ & $\begin{array}{l}\checkmark \text { Raspberry } \\
\text { pi } \\
\checkmark \mathrm{Wi}-\mathrm{Fi}\end{array}$ & $\begin{array}{l}\checkmark \text { Increases } \\
\text { poultry } \\
\checkmark \text { production. } \\
\checkmark \text { optimizes } \\
\text { resource } \\
\text { utilization. } \\
\checkmark \text { Saves time } \\
\checkmark \text { Reduces } \\
\text { human } \\
\text { intervention }\end{array}$ & $\begin{array}{l}\checkmark \text { High cost } \\
\checkmark \text { Maintenance } \\
\text { of labour } \\
\checkmark \text { Wrong } \\
\text { knowledge in } \\
\text { farming } \\
\text { practices. }\end{array}$ & $\begin{array}{l}\checkmark \text { Develop a } \\
\text { poultry } \\
\text { management } \\
\text { system using } \\
\text { low cost } \\
\text { commodity } \\
\text { hardware and } \\
\text { open source } \\
\checkmark \text { software }\end{array}$ & $\begin{array}{l}\checkmark \text { Making } \\
\text { wireless } \\
\text { communica } \\
\text { tion } \\
\text { between } \\
\text { sensor } \\
\text { module and } \\
\text { coordinator } \\
\text {. }\end{array}$ & $\checkmark$ Farming \\
\hline 59 & $\begin{array}{l}\text { Maina (2017) } \\
\text { [59] }\end{array}$ & $\begin{array}{l}\checkmark \text { Livestock } \\
\text { manageme } \\
\text { nt } \\
\checkmark \text { Smart } \\
\text { farming }\end{array}$ & $\begin{array}{l}\checkmark \text { Temperature } \\
\checkmark \text { Size of the cattle } \\
\checkmark \text { Activity of the } \\
\quad \text { cow }\end{array}$ & $\begin{array}{l}\checkmark \text { RFID } \\
\checkmark \text { Raspberry } \\
\quad \text { pi }\end{array}$ & $\begin{array}{l}\checkmark \text { Improve } \\
\text { productivity } \\
\checkmark \text { Can } \\
\text { effectively } \\
\text { detect heat }\end{array}$ & $\begin{array}{l}\checkmark \text { Heat detection } \\
\checkmark \text { Death of } \\
\text { livestock }\end{array}$ & $\begin{array}{l}\checkmark \text { Use prototype } \\
\text { sensor to detect } \\
\text { the activity of } \\
\text { cow. }\end{array}$ & $\begin{array}{l}\checkmark \text { Improving } \\
\text { the system } \\
\text { capability } \\
\text { to detecting } \\
\text { cow } \\
\text { activity in } \\
\text { real time }\end{array}$ & $\checkmark$ Farming \\
\hline 60 & $\begin{array}{l}\text { Memon et al } \\
(2016) \\
{[60]}\end{array}$ & $\begin{array}{l}\checkmark \text { Water } \\
\text { manageme } \\
\text { nt } \\
\checkmark \text { Waste } \\
\text { manageme } \\
\text { nt }\end{array}$ & $\begin{array}{l}\checkmark \text { Temperature } \\
\checkmark \text { Humidity }\end{array}$ & $\begin{array}{l}\checkmark \text { Wi-Fi } \\
\checkmark \text { LAN }\end{array}$ & $\begin{array}{l}\checkmark \text { Provide } \\
\text { required } \\
\text { feed and } \\
\text { water. } \\
\checkmark \text { Exhaust the } \\
\text { excess of } \\
\text { biogas of } \\
\text { animals } \\
\checkmark \text { Surveillance } \\
\text { of the entire } \\
\text { farm }\end{array}$ & $\checkmark$ Stock theft & $\begin{array}{l}\checkmark \text { Develop a } \\
\text { system to } \\
\text { control and } \\
\text { monitor the } \\
\text { farm remotely }\end{array}$ & $\begin{array}{l}\checkmark \text { Improve } \\
\text { the features } \\
\text { of the smart } \\
\text { system }\end{array}$ & $\begin{array}{l}\checkmark \text { Agricultu } \\
\text { re }\end{array}$ \\
\hline
\end{tabular}

\section{DISCUSSION}

In this review we have identified important attributes to analyse the research findings in agriculture and farming processes. We have gathered and analyzed data by using 60 recent scientific articles. Our survey shows the most researched sub-verticals are water management, crop management and smart farming. Water management is the most researched sub-vertical for the last few years as most countries mainly focus on the utilization of water resources due to its lack of abundance [61]. Irrigation patterns in agriculture influence crop production making irrigation management a central focus to increase productivity [8], [10]. The second most considered sub-vertical is crop management due to the importance of producing food for a growing global population. It is important to manage the quality, quantity and effectiveness of the agricultural production for sustainability [13]. Although a study [18] discussed that the widely used sensor data collections for measurements are soil conditions as $\mathrm{pH}$ and humidity, as per our analysis it shows environmental temperature followed by humidity and soil moisture are the most commonly measured data.

IoT can further be defined as a fusion of heterogeneous networks including chip technology that scopes gradually more and more, expanding due to the rapid growth of Internet applications such as logistics, agriculture, smart community, intelligent transposition, control and tracking systems. According to researchers' analysis, in 2020 IoT objects will be semi-intelligent and an important part of human social life [46]. As analyzed in our review Wi-Fi,mobile technology are the technologies which have a wide range of demand in agriculture and farming domain to monitor land and water resources in contrast to other technologies [33], [35].
Although our results demonstrate the results in such a way, a study [62] analyzed that use of RFID, a Wireless Sensor Network (WSN) technology that can be effectively used to increase the crop production to meet the growing needs of the increasing population. In developing countries with limited Internet speed, the other IoT technologies utilised rather than Wi-Fi include Low-Power, Short-Range IoT Networks, lowrate wireless PAN (LoRaWAN) or Low-Power and WideArea Networks.

Further research [61] shows that WSN is used in many applications such as health monitoring, agriculture, environmental monitoring, and military applications whereas our study demonstrates the agriculture sector using IoT in and farming sector using IoT. Our observations show that Agriculture is the primary source of income in developing countries, such as India with the sizeable geographical area when comparing with other countries [9].

Most of the research studies have performed on water management by monitoring such environmental parameters as temperature, humidity and soil moisture [1], [3], [5], [19], [25]. Many of the findings have focused on better water utilization, reduction inhuman intervention and the cost of production [18], [27]. Future research could draw more attention to further automate current processes in waste management, smart lightening and pest controlling subverticals by reducing existing drawbacks since it has received the least research attention in the considered period. Fog computing, as an innovation with cross over any barrier between remote data centres and IoT devices, should be considered in future IoT analysis [63], [64], [65], [66], [67]. While IoT has solved many issues related to agriculture and farming there are limitations that we need to consider. Lack of 
interoperability and compatibility in devices, network flexibility issues when more devices are connecting, and sensor lifetime is some of the limitations to be addressed in future research.

This study has found that industry 4.0 in agriculture focuses on IoT aspects transforming the production capabilities including the agricultural domain. This study has [68] considered soil quality, irrigation levels, weather, the presence of insects and pests as sensor data. Some of the significant aspects they have been researched are the driver's assistance to optimise routes and shorten harvesting and crop treatment while reducing fuel consumption CISCO [69]. Producing enough food for the entire world is a big challenge since the global population is rapidly changing as well as climate change and labour shortage. Currently researchers have focused more on robotics to address these problems. A growing number of researchers and companies have focused on Robotics and Artificial Intelligence (AI) to weeding by reducing the amount of herbicide used by farmers.

In contrast to edge computing, cloud computing requires a high-speed internet connection with sending and retrieving data from the cloud. As the process involves transferring and receiving data from the cloud, the process is time-consuming. Since the data capacity is higher than bandwidth, it is always essential to process data locally instead of sending data to the cloud. Edge computing is more efficient than cloud processing when processing data since the capacity doubles faster than the bandwidth doubles [70]. Since IoT uses sensor data collection for decision making, to process collected data, the cloud, or the edge based can be used on the system requirements.

Still, there are some challenges associated with IoT system deployment. Connecting so many devices to the IoT network is the biggest challenge in the future following lack of technical knowledge among farmers, current centralised architecture to support IoT systems is not much advanced as the growth of the network, centralised systems will turn into a bottleneck. Moreover, sensor battery capacity and lifetime and sensor data storage also more concentrated when IoT system deployment. Smart farming is the association with new advancements in technologies and the different crop and livestock, agriculture and farming in the digital age. Smart farming can deliver agriculture more beneficial for the farmer. This is because decreasing input resources will save farmers' money and labour, and hence, will increase reliability [71] and business outcome [72], [73].

Furthermore, studying diverse approaches for fog computing structure [63], decision making using prediction or pattern analysis [74], [75], [76], big data databases [77] could be an exciting way to make the Internet of Things (IoT) into the future dominating technology.

This survey will fill the gap by the identification of the different IoT sub-verticals and data collections for the measurements in the agriculture and farming process. Results are clearly showing that most considered sub-verticals and data collections for measurements in the field of agriculture and farming. Our study also indicates the technologies used for IoT application development in the reviewed period. To summarise this survey, this has broader knowledge about IoT applications developed for automating the agriculture and farming process. Moreover, this study identifies most considered sub-verticals, collected sensor data and technologies for the development of IoT based applications in agriculture and farming sector towards the significant improvement of the business.

Table II shows the other necessary data collection criteria which were not included in all studies.

TABLE II. IMPORTANT DATA INCLUSION CRITERIA FOR FUTURE IOT STUDIES

\begin{tabular}{|c|c|c|c|}
\hline Criteria & Information to be Collected in IoT Domain & Addressed in this Review & $\begin{array}{c}\text { To be Addressed in Future } \\
\text { Research }\end{array}$ \\
\hline IoT Sub Verticals & What are the sub-areas addressed? & $\checkmark$ & $\checkmark$ \\
\hline Technologies Used & $\begin{array}{l}\text { Used technologies to develop or to solve } \\
\text { problems. }\end{array}$ & $\checkmark$ & $\checkmark$ \\
\hline Challenges in Current Approach & $\begin{array}{l}\text { Existing issues and problems in the current } \\
\text { systems and methods. }\end{array}$ & $\checkmark$ & $\checkmark$ \\
\hline Solution for Current Issues & $\begin{array}{l}\text { Proposed solution to solve the issues in the } \\
\text { current problems. }\end{array}$ & $\checkmark$ & $\checkmark$ \\
\hline Drivers of IoT Countries & $\begin{array}{l}\text { What are the novelty and future aspects of the } \\
\text { proposed systems and methods? }\end{array}$ & $\checkmark$ & $\checkmark$ \\
\hline Heterogeneity Aspects & $\begin{array}{l}\text { Are sensors and underlying technologies } \\
\text { uniform or heterogeneous in the system? }\end{array}$ & $\mathrm{x}$ & $\checkmark$ \\
\hline
\end{tabular}




\begin{tabular}{|c|c|c|c|}
\hline System Architecture & $\begin{array}{l}\text { Complex is the adopted IoT architecture, sensors } \\
\text { topology, information about intermediate } \\
\text { gateways }\end{array}$ & $\mathrm{x}$ & $\checkmark$ \\
\hline Data Analysis Methods & $\begin{array}{c}\text { Business intelligence, Artificial Intelligence, } \\
\text { learning algorithms (machine Learning } \\
\text { algorithms, Deep learning), big data } \\
\text { technologies (Hadoop, Spark) and other } \\
\text { protocols } \\
\end{array}$ & $\mathrm{x}$ & $\checkmark$ \\
\hline Observed System & $\begin{array}{c}\text { Size or scale of the observed land or agricultural } \\
\text { domain }\end{array}$ & $\mathrm{x}$ & $\checkmark$ \\
\hline Access to Natural resources & Water resources and weather condition & $\mathrm{x}$ & $\checkmark$ \\
\hline $\begin{array}{l}\text { IoT Security and Threat Solutions } \\
\text { / Protocols }\end{array}$ & $\begin{array}{c}\text { Encryption techniques for IoT data access, } \\
\text { Vulnerable identity (change default passwords), } \\
\text { self-error detection and possible cyber attacks }\end{array}$ & $\mathrm{x}$ & $\checkmark$ \\
\hline Power Supplies & $\begin{array}{l}\text { Battery, AC power, and other protocol to } \\
\text { optimize energy savings }\end{array}$ & $\mathrm{x}$ & $\checkmark$ \\
\hline
\end{tabular}

\section{CONCLUSION}

From our observations from the 60 peer-reviewed publications (2016-2018) in discussing the potential applications of the Internet of Things, it was found that water management is the highest considered IoT sub-vertical followed by crop management, smart farming, livestock management, and irrigation management with the same percentage. As per the observation, the most critical sensor data collection for the measurement is environmental temperature, environmental humidity and also there are some other such sensor data also gathered for IoT applications as soil moisture and soil $\mathrm{pH}$. Wi-Fi has the highest demand of usage in agriculture and farming industry, followed by mobile technology. Other technologies as ZigBee, RFID, Raspberry pi, WSN, Bluetooth, LoRa and GPRS have less demand in the agriculture and farming sectors. When compared to the agricultural sector, farming industry has a lesser percentage amount using IoT for the automation. This survey could be useful for researchers for finding new ways and solution to challenge in the current agricultural era and for agricultural and farming industries to make the automation process more effective and efficient, consequently, to obtain the good businesses outcome.

\section{AUTHORS' PROFILE}

R.M., S.W., and M.N.H. conceived the study idea and developed the analysis plan. R.M. and S.W. analyzed the data and wrote the initial paper.

M.N.H. helped to prepare the figures and tables and finalizing the manuscript. R.M. completed the final editing and figures of the manuscript. All authors read the manuscript.

\section{REFERENCES}

[1] R. Venkatesan and A. Tamilvanan, "A sustainable agricultural system using IoT," in International Conference on Communication and Signal Processing (ICCSP), 2017.
[2] G. Arvind and V. Athira and H. Haripriya and R. Rani and S. Aravind, "Automated irrigation with advanced seed germination and pest control," in IEEE Technological Innovations in ICT for Agriculture and Rural Development (TIAR), 2017.

[3] W. Zhao and S. Lin and J. Han and R. Xu and L. Hou, "Design and Implementation of Smart Irrigation System Based on LoRa," in IEEE Globecom Workshops (GC Wkshps), 2017.

[4] S. Sagar and G. Kumar and L. Xavier and S. Sivakumar and R. Durai, "Smart irrigation system with flood avoidance technique," in Third International Conference on Science Technology Engineering \& Management (ICONSTEM), 2017.

[5] S. Saraf and D. Gawali, "IoT based smart irrigation monitoring and controlling system," in 2nd IEEE International Conference on Recent Trends in Electronics, Information \& Communication Technology (RTEICT), 2017.

[6] Rama Chidambaram RM and Vikas Upadhyaya, "Automation in drip irrigation using IOT devices," in Fourth International Conference on Image Information Processing (ICIIP), 2017.

[7] S. Vaishali and S. Suraj and G. Vignesh and S. Dhivya and S. Udhayakumar, "Mobile integrated smart irrigation management and monitoring system using IOT," in International Conference on Communication and Signal Processing (ICCSP), 2017.

[8] M. Rajkumar and S. Abinaya and V. Kumar, "Intelligent irrigation system-An IOT based approach," in International Conference on Innovations in Green Energy and Healthcare Technologies (IGEHT), 2017.

[9] A. Rau and J. Sankar and A. Mohan and D. Das Krishna and J. Mathew, "IoT based smart irrigation system and nutrient detection with disease analysis," in IEEE Region 10 Symposium (TENSYMP), 2017.

[10] Sanket Salvi and Pramod Jain S.A and Sanjay H.A and Harshita T.K and M. Farhana and Naveen Jain and Suhas M V, "Cloud based data analysis and monitoring of smart multi-level irrigation system using IoT," in International Conference on I-SMAC (IoT in Social, Mobile, Analytics and Cloud) (I-SMAC), 2017.

[11] P. Sureephong and P. Wiangnak and S. Wicha, "The comparison of soil sensors for integrated creation of IOT-based Wetting front detector (WFD) with an efficient irrigation system to support precision farming," in International Conference on Digital Arts, Media and Technology (ICDAMT), 2017.

[12] S. Rajeswari and K. Suthendran and K. Rajakumar, "A smart agricultural model by integrating IoT, mobile and cloud-based big data 
analytics," in International Conference on Intelligent Computing and Control (I2C2), 2017.

[13] P. Patil and V. Sachapara, "Providing smart agricultural solutions/techniques by using Iot based toolkit," in International Conference on Trends in Electronics and Informatics (ICEI), 2017.

[14] S. Pooja and D. Uday and U. Nagesh and S. Talekar, "Application of MQTT protocol for real time weather monitoring and precision farming," in International Conference on Electrical, Electronics, Communication, Computer, and Optimization Techniques (ICEECCOT), 2017.

[15] O. Pandithurai and S. Aishwarya and B. Aparna and K. Kavitha, "Agrotech: A digital model for monitoring soil and crops using internet of things (IOT)," in Third International Conference on Science Technology Engineering \& Management (ICONSTEM), 2017.

[16] A. Roselin and A. Jawahar, "Smart agro system using wireless sensor networks," in International Conference on Intelligent Computing and Control Systems (ICICCS), 2017.

[17] P. Rekha and V. Rangan and M. Ramesh and K. Nibi, "High yield groundnut agronomy: An IoT based precision farming framework," in IEEE Global Humanitarian Technology Conference (GHTC), 2017.

[18] R. Maia and I. Netto and A. Tran, "Precision agriculture using remote monitoring systems in Brazil," in IEEE Global Humanitarian Technology Conference (GHTC), 2017.

[19] Z. Ahmad and M. Pasha and A. Ahmad and A. Muhammad and S Masud and M. Schappacher and A. Sikora, "Performance evaluation of IEEE 802.15.4-compliant smart water meters for automating large-scale waterways," in 9th IEEE International Conference on Intelligent Data Acquisition and Advanced Computing Systems: Technology and Applications (IDAACS), 2017.

[20] M. Mekala and P. Viswanathan, "A novel technology for smart agriculture based on IoT with cloud computing," in International Conference on I-SMAC (IoT in Social, Mobile, Analytics and Cloud) (ISMAC), 2017.

[21] D. Qi and G. Lu and X. Dai, "Design of Urban Greening Intelligent Monitoring System Based on Internet of Things Technology," in 9th International Conference on Intelligent Human-Machine Systems and Cybernetics (IHMSC), 2017.

[22] Z. Li and J. Wang and R. Higgs and L. Zhou and W. Yuan, "Design of an Intelligent Management System for Agricultural Greenhouses Based on the Internet of Things," in IEEE International Conference on Computational Science and Engineering (CSE) and IEEE International Conference on Embedded and Ubiquitous Computing (EUC), 2017.

[23] C. Cambra and S. Sendra and J. Lloret and L. Garcia, "An IoT serviceoriented system for agriculture monitoring," in IEEE International Conference on Communications (ICC), 2017.

[24] N. Ananthi and J. Divya and M. Divya and V. Janani, "IoT based smart soil monitoring system for agricultural production," in IEEE Technological Innovations in ICT for Agriculture and Rural Development (TIAR), 2017.

[25] S. Prathibha and A. Hongal and M. Jyothi, "IOT Based Monitoring System in Smart Agriculture," in International Conference on Recent Advances in Electronics and Communication Technology (ICRAECT), 2017.

[26] P. Padalalu and S. Mahajan and K. Dabir and S. Mitkar and D. Javale, "Smart water dripping system for agriculture/farming," in 2nd International Conference for Convergence in Technology (I2CT), 2017.

[27] J. Guruprasadh and A. Harshananda and I. Keerthana and Rachana and K. Krishnan and M. Rangarajan and S. Sathyadevan, "Intelligent soil quality monitoring system for judicious irrigation," in International Conference on Advances in Computing, Communications and Informatics (ICACCI), 2017.

[28] S. Athani and C. Tejeshwar and M. Patil and P. Patil and R. Kulkarni, "Soil moisture monitoring using IoT enabled arduino sensors with neural networks for improving soil management for farmers and predict seasonal rainfall for planning future harvest in North Karnataka-India," in International Conference on I-SMAC (IoT in Social, Mobile, Analytics and Cloud) (I-SMAC), 2017.
[29] P. Rajalakshmi and S. Devi Mahalakshmi, "IOT based crop-field monitoring and irrigation automation," in 10th International Conference on Intelligent Systems and Control (ISCO), 2016.

[30] A. Imteaj and T. Rahman and M. Hossain and S. Zaman, "IoT based autonomous percipient irrigation system using raspberry $\mathrm{Pi}$, " in 19th International Conference on Computer and Information Technology (ICCIT), 2016

[31] H. Biradar and L. Shabadi, "Review on IOT based multidisciplinary models for smart farming," in 2nd IEEE International Conference on Recent Trends in Electronics, Information \& Communication Technology (RTEICT), 2017.

[32] M. bin Ismail and N. Thamrin, "IoT implementation for indoor vertical farming watering system," in International Conference on Electrical, Electronics and System Engineering (ICEESE), 2017.

[33] Amandeep and A. Bhattacharjee and P. Das and D. Basu and S. Roy and S. Ghosh and S. Saha and S. Pain and S. Dey and T. Rana, "Smart farming using IOT", 2017 8th IEEE Annual Information Technology," in Electronics and Mobile Communication Conference (IEMCON), 2017.

[34] R. Dolci, "IoT Solutions for Precision Farming and Food Manufacturing: Artificial Intelligence Applications in Digital Food," in IEEE 41st Annual Computer Software and Applications Conference (COMPSAC), 2017.

[35] V. Gokul and S. Tadepalli, "Implementation of smart infrastructure and noninvasive wearable for real time tracking and early identification of diseases in cattle farming using IoT," in International Conference on ISMAC (IoT in Social, Mobile, Analytics and Cloud) (I-SMAC), 2017.

[36] C. Yoon and M. Huh and S. Kang and J. Park and C. Lee, "Implement smart farm with IoT technology," in 20th International Conference on Advanced Communication Technology (ICACT), 2018.

[37] M. Rajkumar and S. Abinaya and V. Kumar, "Intelligent irrigation system-An IOT based approach," in International Conference on Innovations in Green Energy and Healthcare Technologies (IGEHT), 2017.

[38] S. Ruengittinun and S. Phongsamsuan and P. Sureeratanakorn, "Applied internet of thing for smart hydroponic farming ecosystem (HFE)," in 10th International Conference on Ubi-media Computing and Workshops (Ubi-Media), 2017.

[39] A. Ezhilazhahi and P. Bhuvaneswari, "IoT enabled plant soil moisture monitoring using wireless sensor networks," in Third International Conference on Sensing, Signal Processing and Security (ICSSS), 2017.

[40] C. Yoon and M. Huh and S. Kang and J. Park and C. Lee, "Implement smart farm with IoT technology," in 20th International Conference on Advanced Communication Technology (ICACT), 2018.

[41] S. Takekar and S. Takekar, "Plant and taste to reap with Internet of Things implementation of IoT in agriculture to make it a parallel industry," in International Conference on I-SMAC (IoT in Social, Mobile, Analytics and Cloud) (I-SMAC), 2017.

[42] P. Tanmayee, "Rice crop monitoring system-A lot based machine vision approach," in International Conference on Nextgen Electronic Technologies: Silicon to Software (ICNETS2), 2017.

[43] A. Moon and J. Kim and J. Zhang and S. Son, "Lossy compression on IoT big data by exploiting spatiotemporal correlation," in IEEE High Performance Extreme Computing Conference (HPEC), 2017.

[44] Z. Li and J. Wang and R. Higgs and L. Zhou and W. Yuan, "Design of an Intelligent Management System for Agricultural Greenhouses Based on the Internet of Things," in IEEE International Conference on Computational Science and Engineering (CSE) and IEEE International Conference on Embedded and Ubiquitous Computing (EUC), 2017.

[45] D. Sreekantha and Kavya A.M., "Agricultural crop monitoring using IOT - a study," in 11th International Conference on Intelligent Systems and Control (ISCO), 2017.

[46] S. Rajendrakumar and Rajashekarappa and V. Parvati and B. Parameshachari and K. Soyjaudah and R. Banu, "An intelligent report generator for efficient farming," in International Conference on Electrical, Electronics, Communication, Computer, and Optimization Techniques (ICEECCOT), 2017. 
[47] P. Padalalu and S. Mahajan and K. Dabir and S. Mitkar and D. Javale, "Smart water dripping system for agriculture/farming," in 2nd International Conference for Convergence in Technology (I2CT), 2017.

[48] D. Ferreira and P. Corista and J. Giao and S. Ghimire and J. Sarraipa and R. Jardim-Goncalves, "Towards smart agriculture using FIWARE enablers," in International Conference on Engineering, Technology and Innovation (ICE/ITMC), 2017.

[49] T. Okayasu and A. Nugroho and A. Sakai and D. Arita and T. Yoshinaga and R. Taniguchi and M. Horimoto and E. Inoue and Y. Hirai and M. Mitsuoka, "Affordable field environmental monitoring and plant growth measurement system for smart agriculture," in Eleventh International Conference on Sensing Technology (ICST), 2017.

[50] B. Bellini and A. Amaud, "A $5 \mu$ ? wireless platform for cattle heat detection," in IEEE 8th Latin American Symposium on Circuits \& Systems (LASCAS), 2017.

[51] C. Cambra and S. Sendra and J. Lloret and L. Garcia, "An IoT serviceoriented system for agriculture monitoring," in IEEE International Conference on Communications (ICC), 2017.

[52] J. Vaughan and P. Green and M. Salter and B. Grieve and K. Ozanyan, "Floor sensors of animal weight and gait for precision livestock farming," in IEEE SENSORS, 2017.

[53] W. Vernandhes and N. Salahuddin and A. Kowanda and S. Sari, "Smart aquaponic with monitoring and control system based on iot," in Second International Conference on Informatics and Computing (ICIC), 2017.

[54] G. Raghudathesh and D. Deepak and G. Prasad and A. Arun and R. Balekai and V. Yatnalli and S. Lata and B. Kumar, "Iot based intelligent poultry management system using Linux embedded system," in International Conference on Advances in Computing, Communications and Informatics (ICACCI), 2017.

[55] C. wa Maina, "IoT at the grassroots-Exploring the use of sensors for livestock monitoring," in IST-Africa Week Conference (IST-Africa), 2017.

[56] K. Krishna and O. Silver and W. Malende and K. Anuradha, "Internet of Things application for implementation of smart agriculture system," in International Conference on I-SMAC (IoT in Social, Mobile, Analytics and Cloud) (I-SMAC), 2017.

[57] M. Hunain Memon, "Internet of Things (IoT) Enabled Smart Animal Farm," in Computing for Sustainable Global Development (INDIACom), 2016 3rd International Conference, 2016.

[58] G. Suciu and O. Fratu and A. Vulpe and C. Butca and V. Suciu, "IoT agro-meteorology for viticulture disease warning," in IEEE International Black Sea Conference on Communications and Networking (BlackSeaCom), 2016.

[59] N. Putjaika and S. Phusae and A. Chen-Im and P. Phunchongharn and K. Akkarajitsakul, "A control system in an intelligent farming by using arduino technology," in Fifth ICT International Student Project Conference (ICT-ISPC), 2016.

[60] S. Prathibha and A. Hongal and M. Jyothi, "IOT Based Monitoring System in Smart Agriculture," in International Conference on Recent Advances in Electronics and Communication Technology (ICRAECT), 2017.

[61] Rekha B Venkatapur and S Nikitha, "Review on Closed Loop Automated Irrigation System," The Asian Review of Civil Engineering, vol. 6, pp. 9-14, 2017.

[62] K. Bidua and C. Patel, "Internet of Things and Cloud Computing for Agriculture in India," International Journal of Innovative and Emerging Research in Engineering, vol. 2, pp. 27-30, 2015.
[63] B. N. B. Ekanayake and M. N. Halgamuge and A. Syed, "Review: Security and Privacy Issues of Fog Computing for the Internet of Things (IoT)," Lecture Notes on Data Engineering and Communications Technologies Cognitive Computing for Big Data Systems Over IoT, Frameworks, Tools and Applications, Springer, vol. 14, p. Chapter 7, 2018.

[64] J. Gubbi and R. Buyya and S. Marusic and M Palaniswami, "Internet of Things (IoT): A vision, architectural elements, and future directions," Future Generation Computer Systems, vol. 29, no. 7, pp. 1645-1660, 2013.

[65] Ashkan Yousefpour and Genya Ishigaki and Riti Gour and Jason P. Jue, "On Reducing IoT Service Delay via Fog Offloading," IEEE Internet of Things Journal, Vols. 998 - 1010, no. 2, p. 5, 2018.

[66] Shigen Shen and Longjun Huang and Haiping Zhou and Shui Yu and En Fan and Qiying Cao, "Multistage Signaling Game-Based Optimal Detection Strategies for Suppressing Malware Diffusion in Fog-CloudBased IoT Networks," IEEE Internet of Things Journal, vol. 5, no. 2, pp. 1043 - 1054, 2018.

[67] Shuang Zhao and Yang Yang and Ziyu Shao and Xiumei Yang and Hua Qian and Cheng-Xiang Wang, "FEMOS: Fog-Enabled Multitier Operations Scheduling in Dynamic Wireless Networks," IEEE Internet of Things Journal, vol. 5, no. 2, pp. 1169 - 1183, 2018.

[68] [Online]. Available: https://ec.europa.eu/growth/tools-databases/ dem/ monitor/content/industry-40-agriculture-focus-iot-aspects.

[69] [Online]. Available: https://newsroom.cisco.com/feature-content?type $=$ webcontent\&articleId $=1870277$.

[70] K. Dolui and S. K. Datta, "Comparison of edge computing implementations: Fog computing, cloudlet and mobile edge computing," Global Internet of Things Summit (GIoTS), 2017.

[71] A. Walter and R. Finger and R. Huber and N. Buchmanna, "Opinion: Smart farming is key to developing sustainable agriculture," Proc Natl Acad Science, vol. 114, p. 6148-6150, 2017.

[72] E. Hakanen, R. Rajala, "Material intelligence as a driver for value creation in IoT-enabled business ecosystems", Journal of Business \& Industrial Marketing, September 2018.

[73] T. Osmonbekov, W. J. Johnston, "Adoption of the Internet of Things technologies in business procurement: impact on organizational buying behavior", Journal of Business \& Industrial Marketing, September 2018.

[74] A. Gupta, A. Mohammad, A. Syed, and M. N. Halgamuge, "A Comparative Study of Classification Algorithms using Data Mining: Crime and Accidents in Denver City the USA", International Journal of Advanced Computer Science and Applications (IJACSA), Volume 7, Issue 7, pp 374 - 381, August 2016.

[75] C. Wanigasooriya, M. N. Halgamuge, A. Mohamad, "The Analyzes of Anticancer Drug Sensitivity of Lung Cancer Cell Lines by Using Machine Learning Clustering Techniques", International Journal of Advanced Computer Science and Applications (IJACSA), Volume 8, No 9, September 2017.

[76] A. Singh, M. N. Halgamuge, R. Lakshmiganthan, "Impact of Different Data Types on Classifier Performance of Random Forest, Naïve Bayes, and k-Nearest Neighbors Algorithms", International Journal of Advanced Computer Science and Applications (IJACSA), Volume 8, No 12, pp 1-10, December 2017.

[77] V. Vargas, A. Syed, A. Mohammad, and M. N. Halgamuge, "Pentaho and Jaspersoft: A Comparative Study of Business Intelligence Open Source Tools Processing Big Data to Evaluate Performances", International Journal of Advanced Computer Science and Applications (IJACSA), Volume 7, Issue 10, pp 20-29, November 2016. 